\title{
Composite Spectral Energy Distributions and Infrared-Optical Colors of Type 1 and Type 2 Quasars
}

\author{
Ryan C. Hickox ${ }^{1}$ (1), Adam D. Myers ${ }^{2,3}$, Jenny E. Greene ${ }^{4}$, \\ Kevin N. Hainline ${ }^{5}$ (i), Nadia L. Zakamska ${ }^{6}$ (i), and Michael A. DiPompeo ${ }^{1}$ \\ ${ }^{1}$ Department of Physics and Astronomy, Dartmouth College, 6127 Wilder Laboratory, Hanover, NH 03755, USA; ryan.c.hickox@dartmouth.edu \\ 2 Department of Physics and Astronomy, University of Wyoming, Laramie, WY 82071, USA \\ ${ }^{3}$ Max-Planck-Institut für Astronomie, Königstuhl 17, D-69117 Heidelberg, Germany \\ ${ }^{4}$ Department of Astrophysics, Princeton University, Princeton, NJ 08544-1001, USA \\ ${ }^{5}$ Steward Observatory, University of Arizona, 933 North Cherry Avenue, Tucson, AZ 85721, USA \\ ${ }^{6}$ Department of Physics and Astronomy, Johns Hopkins University, Bloomberg Center, 3400 North Charles Street, Baltimore, MD 21218, USA \\ Received 2016 November 29; revised 2017 August 13; accepted 2017 September 11; published 2017 October 30
}

\begin{abstract}
We present observed mid-infrared and optical colors and composite spectral energy distributions (SEDs) of type 1 (broad-line) and 2 (narrow-line) quasars selected from Sloan Digital Sky Survey (SDSS) spectroscopy. A significant fraction of powerful quasars are obscured by dust and are difficult to detect in optical photometric or spectroscopic surveys. However, these may be more easily identified on the basis of mid-infrared (MIR) colors and SEDs. Using samples of SDSS type 1 and 2 matched in redshift and [O III] luminosity, we produce composite restframe $0.2-15 \mu \mathrm{m}$ SEDs based on SDSS, UKIDSS, and Wide-field Infrared Survey Explorer photometry and perform model fits using simple galaxy and quasar SED templates. The SEDs of type 1 and 2 quasars are remarkably similar, with the differences explained primarily by the extinction of the quasar component in the type 2 systems. For both types of quasar, the flux of the active galactic nucleus (AGN) relative to the host galaxy increases with AGN luminosity $\left(L_{[\mathrm{O} \text { III] }}\right)$ and redder observed MIR color, but we find only weak dependencies of the composite SEDs on mechanical jet power as determined through radio luminosity. We conclude that luminous quasars can be effectively selected using simple MIR color criteria similar to those identified previously (W1-W2 > 0.7; Vega), although these criteria miss many heavily obscured objects. Obscured quasars can be further identified based on optical-IR colors (for example, $(u-\mathrm{W} 3[\mathrm{AB}])>1.4(\mathrm{~W} 1-\mathrm{W} 2$ [Vega] $)+3.2)$. These results illustrate the power of large statistical studies of obscured quasars selected on the basis of MIR and optical photometry.
\end{abstract}

Key words: galaxies: active - infrared: galaxies - quasars: general - surveys

Supporting material: machine-readable table

\section{Introduction}

Studies of optically luminous quasars have yielded remarkable insights into the growth of supermassive black holes (BHs) over cosmic time (see Alexander \& Hickox 2012, for a review). Analyses of the broadband spectral energy distributions (SEDs) of unobscured ("type 1") quasars have elucidated the physics of BH accretion (Elvis et al. 1994; Richards et al. 2006; Kelly et al. 2010), and surveys in the soft X-rays and optical have shown that quasar activity (and thus BH growth) peaks at early cosmic times $(z \sim 2-3$; e.g., Croom et al. 2004; Hasinger et al. 2005; Richards et al. 2005; Fan et al. 2006). Quasar clustering measurements (e.g., Porciani et al. 2004; Croom et al. 2005; Coil et al. 2007; Myers et al. 2007; Shen et al. 2007; da Ângela et al. 2008; Padmanabhan et al. 2009; Ross et al. 2009; Krumpe et al. 2010; DiPompeo et al. 2016, 2017) suggest that the processes that fuel rapid BH growth are tied to the buildup of large-scale structure (e.g., Hopkins et al. 2008; Croton 2009; Conroy \& White 2013) and indicate that quasars may play a role in regulating star formation and in the emergence of the red galaxy population (e.g., Brown et al. 2008; Coil et al. 2008; Tinker \& Wetzel 2010; Thacker et al. 2014; Vogelsberger et al. 2014; Schaye et al. 2015).

These many successes reveal only part of the story. It has long been known that $\mathrm{BH}$ growth can occur behind large columns of gas and dust (e.g., Setti \& Woltjer 1989; Comastri et al. 1995). Such obscured ("type 2") active galactic nuclei (AGNs) can be identified from narrow optical emission lines (Zakamska et al. 2003, 2004, 2005; Reyes et al. 2008; Yuan et al. 2016), radio luminosity (e.g., McCarthy 1993; MartínezSansigre et al. 2006; Seymour et al. 2007; Wilkes et al. 2013), or X-ray properties (e.g Alexander et al. 2001; Stern et al. 2002; Treister et al. 2004; Vignali et al. 2006, 2009; Del Moro et al. 2014). In addition, pioneering work with the Spitzer Space Telescope demonstrated that obscured quasars have similar mid-infrared (MIR) SEDs to their unobscured counterparts but are dominated by host galaxy light in the optical (Lacy et al. 2004; Rowan-Robinson et al. 2005; Stern et al. 2005; Alonso-Herrero et al. 2006; Martínez-Sansigre et al. 2006; Polletta et al. 2006; Hickox et al. 2007; Donley et al. 2008; Lacy et al. 2013, 2015; Dai et al. 2014). Consequently, obscured quasars can be most efficiently selected in the MIR, as they appear very red in the IRAC [3.6] - [4.5] color, characteristic of the "hot" MIR SED that is evident in broadline quasars (e.g., Richards et al. 2006; Assef et al. 2010, hereafter R06 and A10, respectively). However, in contrast to their unobscured counterparts, obscured quasars have very red optical-IR colors, due to extinction of the nuclear emission in the rest-frame optical and UV (e.g., Polletta et al. 2006; Hickox et al. 2007). Spitzer therefore unveiled significant samples of obscured quasars (see Section 1 of Hickox et al. 2011), and 
MIR studies find roughly equal numbers of obscured and unobscured quasars (e.g., Hickox et al. 2007; Assef et al. 2015).

Obscured quasars thus represent a large fraction of the massive $\mathrm{BH}$ growth in the universe, but it remains unclear whether the obscuration is due to a dusty torus intrinsic to the central engine (as posited in the "unified model" of AGNs; e.g., Antonucci 1993; Urry \& Padovani 1995), to larger-scale clouds in the host galaxy (e.g., Sanders et al. 1988; Page et al. 2004; Goulding et al. 2012; Chen et al. 2015), or to material distributed over a wide range of scales. It is also unclear whether obscured quasars eventually evolve into unobscured objects as they blow away their surrounding dust, as posited by some evolutionary models (e.g., Sanders et al. 1988; Di Matteo et al. 2005; Hopkins et al. 2008). To answer these questions, we require significant statistical samples of obscured quasars, but samples selected with Spitzer are small, numbering 1000 at most relative to $>10^{6}$ known unobscured quasars (e.g., Richards et al. 2009). Thus, our understanding of obscured quasars as a cosmological population remained comparatively limited.

The completion of the Wide-field Infrared Survey Explorer (WISE; Wright et al. 2010) survey of the full sky has allowed us to make dramatic progress in statistical studies of AGNs (e.g., Mor \& Trakhtenbrot 2011; Donoso et al. 2012; Edelson \& Malkan 2012; Eisenhardt et al. 2012; Ichikawa et al. 2012; Mateos et al. 2012; Plotkin et al. 2012; Sajina et al. 2012; Stern et al. 2012; Assef et al. 2013; Geach et al. 2013; DiPompeo et al. 2014, 2015, 2016, 2017; Assef et al. 2015, 2017; Secrest et al. 2015). In particular, WISE enables us to detect and characterize hundreds of thousands of obscured quasars, increasing the sizes of obscured quasar samples by two orders of magnitude and allowing the first large statistical studies of their properties.

In this paper we use WISE photometry to explore the MIR colors and composite broadband SEDs of obscured and unobscured quasars selected from the Sloan Digital Sky Survey (SDSS), using photometry from WISE, SDSS, the UKIRT Infrared Deep Sky Survey (UKIDSS), the Two Micron All Sky Survey (2MASS), and the Galaxy Evolution Explorer $(G A L E X)$. This work builds on several recent studies that have developed various AGN color selection criteria using WISE photometry (e.g., Jarrett et al. 2011; Mateos et al. 2012; Stern et al. 2012). Here we focus specifically on rare, luminous obscured quasars identified using optical spectroscopy, for which the largest sample consists of the type 2 quasars from SDSS (Zakamska et al. 2003; Reyes et al. 2008; Yuan et al. 2016). We compute the optical and MIR colors and composite SED of these obscured quasars and compare them to optically selected unobscured quasars that are matched in redshift and luminosity, with the aim of developing photometric criteria that will allow us to select large samples of very luminous obscured and unobscured quasars for future measurements of space densities, clustering, and other statistical studies. We further explore the dependence of quasar SED shapes on physical and observational properties (AGN luminosity, MIR color, and radio-loudness). The paper is organized as follows: In Sections 2 and 3 we introduce our SDSS quasar samples and the SDSS and WISE photometric data set, in Section 4 we compute composite optical-IR SEDs for the two sets of quasars, and in Section 5 we explore photometric selection criteria. In Section 6 we summarize our results and discuss future applications of these results. Throughout the paper we assume a $\Lambda$ CDM cosmology with $H_{0}=70 \mathrm{~km} \mathrm{~s}^{-1} \mathrm{Mpc}^{-1}$, $\Omega_{m}=0.3$, and $\Omega_{\Lambda}=0.7$.

\section{Quasar Samples}

We employ two samples of quasars selected from the spectroscopic database of SDSS. The first sample (QSO 1s) consists of broad-line (type 1) quasars selected for spectroscopy based on their blue optical-UV colors. These objects are spectroscopically confirmed to have broad permitted emission lines indicative of gas moving in the gravitational potential of a $\mathrm{BH}$. The other sample consists of obscured, narrow-line (type 2) QSOs. These were targeted by SDSS based on unusual colors or detections at X-ray or radio wavelengths but were then spectroscopically selected to have luminous emission in the $[\mathrm{O}$ III] $\lambda 5007$ emission line.

Broad-line objects were selected from the SDSS catalog of Shen et al. (2011). This work presents spectroscopic measurements for a well-defined sample of optical- to UV-selected unobscured QSOs from the Seventh Data Release (DR7) of the SDSS (Abazajian et al. 2009). As mentioned above, approximately half of the sources are selected for observation originally because of their blue colors (Richards et al. 2002). The remainder either were targeted by earlier versions of the QSO selection algorithm or arose for some "serendipitous" reason (such as radio or X-ray detection). For our purposes we do not distinguish between sources based on targeting. Shen et al. fit the quasar continuum (including the broad Fe II pseudo-continuum) and model the broad and narrow lines with multi-Gaussian fits. From the Shen et al. catalog we extract the $\sim 19,000$ QSOs that fall in the redshift range of interest, $0.1<z<0.8$ (chosen to match the approximate redshift range of the obscured QSOs described below and to exclude contamination at very low redshift).

As discussed below, we use the [O III] luminosity $\left(L_{[\mathrm{O} \text { III] }}\right.$, a proxy for bolometric AGN luminosity; e.g., LaMassa et al. 2010) to produce matched samples of obscured and unobscured sources. We use $L_{[\mathrm{O} \text { III] }}$ as our primary AGN luminosity indicator because it is readily available for all sources, can be used for both type 1 and 2 quasars, and was produced directly from the spectroscopic analysis from which the samples were selected. However, we note that $L_{[\mathrm{O} \text { III }]}$ is not a perfect indicator of intrinsic AGN power. For example, $L_{[\mathrm{O} \mathrm{III]}}$ is found to correlate only weakly with intrinsic hard X-ray luminosity, which is a more direct measure of the instantaneous power of the central engine (Berney et al. 2015). Further, it is possible for the [O III] flux to be affected by dust extinction in the host galaxy (e.g., Zakamska et al. 2006), and the relationship between $L_{[\mathrm{O} \text { III] }}$ and bolometric luminosity may be complex, depending, for example, on the extent of the emitting narrow-line region (e.g., Hainline et al. 2013, 2014b, 2016a; Husemann et al. 2013).

The type 2 quasars (QSO 2s) were taken from Reyes et al. (2008) and Yuan et al. (2016), which expand the original search of Zakamska et al. (2003). Reyes et al. and Yuan et al. begin with the SDSS spectroscopic database and select targets with emission-line fluxes indicative of photoionization by an AGN, either through diagnostic line ratios (e.g., Baldwin et al. 1981; Kewley et al. 2001) or through the equivalent widths of the $[\mathrm{O} \mathrm{III}]$ or $[\mathrm{Ne} \mathrm{V}] \lambda 3426$ emission lines (e.g., Zakamska et al. 2003; Gilli et al. 2010). They remove type 1 (unobscured) QSOs by eliminating sources with broad 
permitted emission (typically $\mathrm{H} \beta$ ) with line widths that are substantially broader than that observed for [O III] as determined through visual inspection of the line fits. For their high-redshift $(z>0.52)$ sample, Yuan et al. applied an initial limit of $\operatorname{FWHM}(\mathrm{H} \beta)<1000 \mathrm{~km} \mathrm{~s}^{-1}$ to remove broad-line sources; visual inspection of the spectra identified a small number (10) of QSO 2s with $\operatorname{FWHM}(\mathrm{H} \beta)>1000 \mathrm{~km} \mathrm{~s}^{-1}$. After compiling the full QSO 2 catalogs from the Reyes et al. and Yuan et al. catalogs, we follow Reyes et al. and impose a luminosity cut of $L_{[\mathrm{O} \text { III }]}>10^{8.3} L_{\odot}$ for completeness. Unlike the broad-line quasars, which were specifically targeted by the SDSS based on color, the obscured sources were targeted for a variety of reasons, primarily because many were included in spectroscopic follow-up by the serendipitous targeting algorithm. Some had the colors of high-redshift QSOs because of the high equivalent width emission lines, while some had radio or X-ray counterparts; we refer the reader to Reyes et al. (2008) and Yuan et al. (2016) for the full details of the sample selection.

To enable a statistically useful comparison between the unobscured and obscured quasar populations, we define a subset of the full type 1 quasar sample that is selected to have the same distribution as the QSO 2s in bins of width 0.07 in redshift and 0.1 in $\left.\log L_{[\mathrm{O}} \mathrm{II}\right]$. This selection yields 3425 "matched" type 1 quasars. We note that some QSO 1s are matched to multiple QSO 2s (approximately 20\% of the QSO 1s for our main SDSS+UKIDSS+WISE analysis); for these objects the matched QSO 1s are included multiple times in the averaging of the SEDs, so as best to mirror the $z$ and $L_{[\mathrm{O} \text { III] }}$ distributions of the QSO 2s. This matched comparison sample of type 1 quasars will compose the sample referred to as QSO $1 \mathrm{~s}$ for the remainder of the paper. The distributions in $z$ and $L_{[\mathrm{O} \text { III }]}$ for the QSO 1s and 2s are shown in Figure 1.

\section{Photometric Data}

Photometric data for studying SEDs and colors are taken from the SDSS Data Release 9 (DR9; Ahn et al. 2012), the UKIDSS Large Area Survey (LAS; Lawrence et al. 2007), and the AllWISE catalog (Wright et al. 2010). As a cross-check on our SEDs for a limited subset of bright objects, we also make use of UV photometry from the GALEX All-sky Imaging Survey (AIS; Bianchi 2014) and near-IR (NIR) photometry from 2MASS (Skrutskie et al. 2006). Catalog matching was performed using the TOPCAT software (Taylor et al. 2005).

For ugriz photometry we use SDSS model magnitudes (in the $\mathrm{AB}$ system), corrected for Galactic reddening, as obtained from the SpecPhoto database; the $95 \%$ completeness limits for SDSS photometry are 22.0, 22.2, 22.2, 21.3, and 20.5 (AB) for the $u, g, r, i$, and $z$ bands, respectively. Essentially all $(>99.7 \%)$ of the quasars are detected at $>2 \sigma$ significance in each of the SDSS bands, except the $u$ band, for which only $69 \%$ of the QSO 2 s (but $>99.7 \%$ of the QSO 1s) have a significant detection.

WISE photometric data were obtained using the Gator online database to search for all objects in the AllWISE Source Catalog at the positions of the SDSS quasars. While the AllWISE flux limits vary depending on position on the sky, typical magnitude limits at high Galactic latitude are $\sim 17,16$, 12 , and 9 (Vega) in the 3.4, 4.6, 12, and $22 \mu \mathrm{m}$ bands (W1W4, respectively). Of the 3425 QSO $1 \mathrm{~s}$ and 2892 QSO 2s, all but $19(99.4 \%)$ and $143(95.1 \%)$, respectively, have WISE counterparts within $2^{\prime \prime}$. We use observed magnitudes in each of

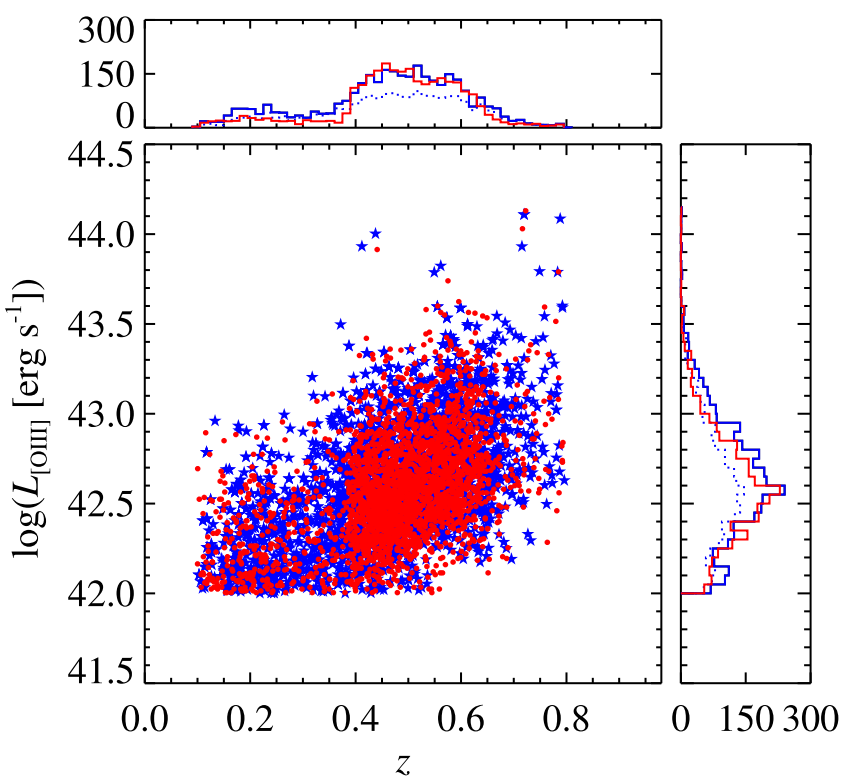

Figure 1. Distribution in redshift and [O III] luminosity for the sample of SDSS type 2 quasars (QSO 2s; red circles) and the sample of type 1 quasars (QSO 1s; blue stars) matched in $z$ and $L_{[\mathrm{O} I I]}$. The dotted blue histograms show the distributions for the full sample of QSO 1s, while the solid blue histograms show the distribution accounting for QSO 1s that were matched in $z$ and $\left.L_{[\mathrm{O}} \mathrm{III}\right]$ to more than one QSO 2, as described in Section 2.

the W1-W4 bands and convert to fluxes using standard conversions from Vega to AB magnitudes. ${ }^{7}$ Among the QSO $1 \mathrm{~s}, 99 \%$ and $93 \%$ have $>2 \sigma$ detections in the W3 and W4 bands, respectively, while the corresponding fractions for QSO 2s are $87 \%$ and $76 \%$.

For NIR photometry, we obtained UKIDSS LAS $Y_{-}, J_{-}, H-$, and $K$-band catalogs from the LAS DR9 database (Lawrence et al. 2012), which has a $5 \sigma$ depth for point sources of $K \approx 18.4$ (Vega). We match to the SDSS positions within $1^{\prime \prime}$. We use Petrosian magnitudes and convert to fluxes using the standard conversion from Vega to $\mathrm{AB}$ magnitudes (Hewett et al. 2006). Of the 3425 QSO 1s and 2892 QSO 2s, 1052 and 860 lie within the $4000 \mathrm{deg}^{2}$ UKIDSS LAS footprint, respectively, and of these, 971 (94\%) and 754 (90\%), respectively, have counterparts within $1^{\prime \prime}$ that have significant detections in all four UKIDSS bands.

Given these high detection fractions in the coverage areas, we focus our primary SED analysis on the quasars (both type 1 and 2) having SDSS, WISE, and UKIDSS detections. However, we also utilize the full SDSS+WISE sample for studies of the color distributions. In general, WISE colors are presented in the Vega system, while colors involving optical magnitudes are presented in the $\mathrm{AB}$ system. To construct SEDs, we compute fluxes at the central wavelengths of the bands for SDSS ugriz $(0.36,0.47,0.62,0.75$, and $0.89 \mu \mathrm{m})$; UKIDSS $Y, J, H$, and $K$ $(1.03,1.25,1.63$, and $2.20 \mu \mathrm{m})$; and WISEW1-W4 (3.4, 4.6, 12 , and $22 \mu \mathrm{m})$.

As a cross-check of our analysis, we also utilize GALEX and 2MASS photometry for a subset of brighter, lower-redshift sources. GALEX AIS sources were taken from the catalog of Bianchi et al. (2011), which reaches $5 \sigma$ depths of $\approx 19.9$ and $\approx 20.8(\mathrm{AB})$ in the far-UV and near-UV bands, respectively. We find that 2558 QSO 1s and 1919 QSO 2s lie within the AIS

\footnotetext{
7 Magnitude offsets, where $\Delta m=m_{\mathrm{AB}}-m_{\mathrm{Vega}}$, are $2.699,3.339,5.174$, and 6.620 in the W1-W4 bands, respectively.
} 
footprint (within $1^{\prime}$ of a GALEX source), and of these, 2549 $(96 \%)$ and $713(37 \%)$ of the QSO 1s and 2s, respectively, are matched to the GALEX positions within $3^{\prime \prime}$. GALEX fluxes are obtained from the Bianchi et al. catalog and corrected for Galactic reddening following Bianchi (2011). We obtain 2MASS photometry from the Point Source Catalog (Cutri et al. 2003), with a depth of $K_{s} \approx 15$ (Vega), and Extended Source Catalog (Skrutskie et al. 2006), with a depth of $K_{s} \approx 14$ (Vega); $1654(48 \%)$ and $300(10 \%)$ of the QSO 1s and 2 s, respectively, are matched to 2 MASS sources within 1.15 . We convert 2MASS magnitudes to monochromatic fluxes following Cohen et al. (2003). The central wavelengths for the GALEX far-UV and near-UV and 2MASS $J, H$, and $K_{s}$ bands are $0.15,0.23,1.24,1.66$, and $2.16 \mu \mathrm{m}$, respectively. The GALEX and 2MASS detection fractions, particularly for the QSO 2s, are a strong function of redshift and rise to $>99 \%$ for QSO 1s and 93\% for QSO 2s for both 2MASS and GALEX if we limit the redshift range to $z<0.2$. We therefore focus our GALEX and 2MASS analysis on quasars at $z<0.2$.

Finally, we utilize radio data to distinguish between quasars that are radio-loud (RL) and radio-quiet (RQ), corresponding to the presence or absence of strong relativistic jets (e.g., Padovani 2016, 2017). We perform a cross-match of the QSO samples to the Faint Images of the Radio Sky at Twenty cm (FIRST; Helfand et al. 2015) and NRAO VLA Sky Survey (NVSS; Condon et al. 1998) catalogs, with point-source detection sensitivities at $20 \mathrm{~cm}$ of $\approx 1$ and $\approx 2.5 \mathrm{mJy}$, respectively. We search for radio counterparts to the QSOs within 1."5 for the FIRST catalog and $10^{\prime \prime}$ for the NVSS catalog; these radii reflect the different angular resolutions of the two surveys $\left(\approx 5^{\prime \prime}\right.$ and $45^{\prime \prime}$, respectively) and are chosen to be the angle at which the frequency of matches approximately reaches that of the background at large separations (the results are insensitive to the precise choice of match radius). We identify a radio counterpart for $723(21 \%)$ of QSO 1s and 1075 (37\%) of QSO 2s. For the $45 \%$ of radio counterparts with both FIRST and NVSS detections, most show good agreement in the fluxes. For $\approx 10 \%$ of cases the NVSS flux is higher by greater than a factor of 2, likely corresponding to additional flux resolved within the larger NVSS beam. For sources with both FIRST and NVSS detections, we adopt the higher value of the flux for the subsequent analysis (broadly following the approach of Zakamska et al. 2004).

For each radio source we calculate the radio power $P_{1.4 \mathrm{GHz}}$ (in $\mathrm{W} \mathrm{Hz}^{-1}$ ), performing a small $K$-correction assuming the typical spectrum of faint $1.4 \mathrm{GHz}$ sources $(\alpha \approx 0.5$, where $S_{\nu} \propto \nu^{-\alpha}$; Prandoni et al. 2006). We select quasars as RL based on a monochromatic $1.4 \mathrm{GHz}$ luminosity threshold of $10^{24} \mathrm{~W}$ $\mathrm{Hz}^{-1}$. This is the approximate luminosity limit for the FIRST survey at $z=0.8$, is characteristic of moderately powerful radio AGNs (e.g., Hickox et al. 2009; Tadhunter 2016), and is above the luminosity for typical star-forming galaxies at low redshift (Kauffmann et al. 2008). Among our samples, 456 (13\%) of the QSO 1s and 600 (21\%) of the QSO 2s are identified as RL. Despite the difference in detection fractions, Zakamska et al. (2004) demonstrate that after accounting for the selection bias toward more radio-bright sources, the intrinsic RL fraction of optically selected type 2 quasars is similar to that of optically selected type $1 \mathrm{~s}$. We note that some QSOs, and particularly QSO 2s, are expected to have lobes that extend up to megaparsec in scale, well beyond our angular matching radius. Thus, sources that have only bright lobe emission with no compact core may be missed by our matching procedure. However, Zakamska et al. (2004) performed a careful matching analysis of the Zakamska et al. (2003) SDSS QSO 2 sample to the FIRST catalog, including a visual examination of the FIRST images for those sources that did not have a counterpart within $3^{\prime \prime}$. Zakamska et al. (2004) found that only a few percent of the QSO 2s had extended emission in the FIRST images but were not matched to a compact core. This small fraction of missed sources indicates that this incompleteness will have a minimal effect on our analysis, which focuses on composite SEDs for the full RL and RQ samples.

\section{Composite Quasar SEDs}

We next derive composite SEDs of the QSO 1 and QSO 2 samples. The composite SEDs allow us to compare the overall physical characteristics of the populations and to model how the selection of these objects will depend on redshift, luminosity, and other parameters.

We use a simple procedure to construct a mean SED for each of the two populations. We include only sources with detections in all 12 SDSS, UKIDSS, and WISE bands, as described in Section 3, which represents 334 QSO 1s and 406 QSO 2s, respectively. Because of the relatively high detection rates in each band (Section 3 ), these criteria are not expected to significantly bias the results toward or away from any particular subsample. We confirm this explicitly by performing the same analysis described here, but including sources with marginal detections for which fluxes are given in the SDSS and WISE catalogs. This particularly allows us to include fluxes from the less sensitive $u$ and $\mathrm{W} 4$ bands that are below the formal detection limit. Including these sources, the results on the composite SEDs are essentially identical, so for the remainder of the paper we focus on sources with robust detections in all bands of interest.

For each object, we determine the fluxes at the rest-frame wavelengths corresponding to the observed photometric bands and then interpolate between these using piecewise power laws (linear in log-log space) to produce a rough SED for each object. We then normalize each object's SED based on the integrated flux in the rest-frame $8-13 \mu \mathrm{m}$. This corresponds roughly to the integrated observed flux between the W3 and W4 WISE bands and so corresponds to the part of the spectrum that has robust flux measurements at the longest wavelengths, where it suffers least from dust attenuation (although, as we note below and discuss in Section 6, obscuration may still have some effect on the AGN SED at these wavelengths). At each wavelength, the logarithms of these interpolated and normalized fluxes are then averaged at each wavelength (eliminating fluxes with a deviation from the mean of $>6 \sigma$ to avoid biasing the average owing to outliers) and the variance computed. We thus produce a final composite SED template for the sources in each subset of QSOs.

\subsection{Comparison of QSO 1 and 2 SEDs}

The average optical-MIR SEDs with the corresponding variance are shown in Figure 2 and tabulated in Table 1. There are clear differences between the SEDs of the QSO 1s and 2s: the QSO 1 SED is very close to flat in $\nu F_{\nu}$, while the QSO 2 SED shows clear features, including a break at $\approx 4000 \AA$ (corresponding to the characteristic break in SEDs of galaxies) and a redder continuum at $>4 \mu \mathrm{m}$. 


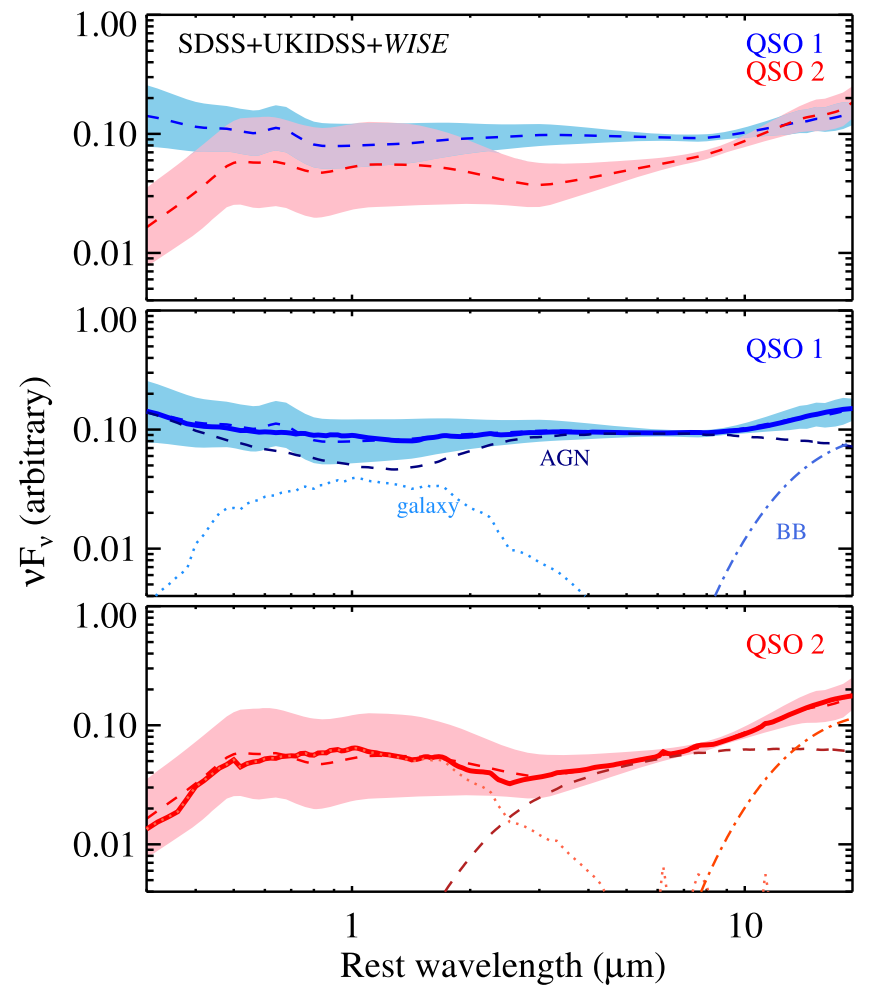

Figure 2. Top panel: normalized composite SEDs for the QSO 1s (blue) and QSO 2s (red), computed as described in Section 4. Each individual quasar SED is normalized by the integrated flux between 8 and $13 \mu \mathrm{m}$ and averaged to create the composite; these are then scaled according to the relative flux of their (unabsorbed) AGN components derived from the SED fitting procedure described in Section 4. Middle and bottom panels: fits to the composite SEDs using a three-component model including host galaxy (dotted lines), AGN (dashed lines), and blackbody (dot-dashed lines) components. Here the dotted line shows the sum of the E and Im galaxy components. Note that the two SEDs show similar ratios of AGN and host galaxy flux, with the primary difference being the reddening of the quasar continuum in the QSO 2s. These best-fit model components are used to make predictions for the observed colors of obscured quasars as a function of redshift, as described in Section 5.

To better understand the differences between these SEDs, we perform fits to the composite SEDs with a simple multicomponent model consisting of quasar continuum (with dust extinction) and a host galaxy, with an additional blackbody component at long wavelengths, as described below. For the quasar SED we use the type 1 quasar template presented in R06 and apply reddening using an extinction curve given by the parameterization of Fitzpatrick (1999). For the host galaxy template we use a linear combination of two empirical galaxy SEDs ( $\mathrm{E}$ and Im, corresponding to old and young stellar populations, respectively) derived by A10. We note that the analysis of A10 also included an intermediate (Sbc) template, but we find that the $\mathrm{E}$ and $\mathrm{Im}$ templates alone are sufficient to capture the full variance of the SEDs and so omit the Sbc template for simplicity. We have also used galaxy SED models computed from the PEGASE stellar evolution code (Fioc \& Rocca-Volmerange 1997), with no significant change in the results. We find that the QSO 1 and 2 SEDs show an excess beyond the 06 quasar SED at $>8 \mu \mathrm{m}$. We model this excess with a simple blackbody continuum, with best-fit temperature $\approx 150 \mathrm{~K}$. We note that the AGN template from A10 does not fit the average SEDs quite as well as the R06 QSO template, but using that AGN template for the full analysis does not
Table 1

Composite QSO 1 and 2 SEDs

\begin{tabular}{lccccc}
\hline \hline & \multicolumn{2}{c}{ Scaled QSO 1 SED } & & \multicolumn{2}{c}{ Scaled QSO 2 SED } \\
\cline { 2 - 3 } \cline { 5 - 6 } $\log _{10}(\lambda(\mu \mathrm{m}))$ & $\left\langle\log _{10}\left(F_{\nu}\right)\right\rangle$ & Scatter $(\mathrm{dex})$ & & $\left\langle\log _{10}\left(F_{\nu}\right)\right\rangle$ & Scatter $(\mathrm{dex})$ \\
\hline-0.650 & -1.420 & 0.254 & & -2.668 & 0.277 \\
-0.625 & -1.445 & 0.269 & & -2.594 & 0.320 \\
-0.600 & -1.436 & 0.272 & & -2.510 & 0.327 \\
-0.575 & -1.417 & 0.271 & & -2.439 & 0.326 \\
-0.550 & -1.395 & 0.266 & & -2.386 & 0.328 \\
\hline
\end{tabular}

Note. The composite SEDs presented here are for the primary sample using SDSS, UKIDSS, and WISE photometry, produced as described in Section 4 and as shown in Figure 2.

(This table is available in its entirety in machine-readable form.)

significantly affect our general conclusions (see Section 4.3 for details).

We perform least-squares fits of this four-component model (two galaxies, absorbed quasar, and blackbody) to the QSO 1 and 2 composite SEDs. To determine the uncertainties in our model fits to these SEDs, we perform bootstrap resampling. Each subset of QSOs is sampled randomly (with replacement), and the composite SEDs are computed and fitted 100 times. The uncertainties in the fit parameters are determined by the variance in the best-fit values among the bootstrap samples.

The fits are shown in Figure 2, and the best-fit parameters and bootstrap uncertainties are given in Table 2. Note that in Figure 2 the SEDs are normalized to have equal flux in the unreddened AGN component, to best illustrate the effects of reddening. The fits to the two SEDs are remarkably similar, with comparable relative normalizations of the quasar and galaxy components (these are within $\approx 20 \%$ ) and blackbody temperatures $\approx 150 \mathrm{~K}$. The main difference is in the reddening of the AGN component. For the QSO 1s the model fits prefer the lower limit of zero extinction, while for the QSO 2s the best fit yields $A_{V} \approx 20$. Another difference is in the normalizations of the blackbody component, which is twice as large for the QSO 2s relative to the QSO 1s. This may indicate that the effects of dust extinction extend well into the MIR, or an extra contribution due to star formation in QSO 2 hosts; we discuss these possibilities in Section 6. The primary conclusion from these model fits is that the QSO 1 and QSO 2 SEDs can be broadly described by the same simple model. We use this model in Section 5 to make predictions for selection of these objects based on optical and MIR photometry.

In Figure 3, we also show the composite SEDs including GALEX and 2MASS data for QSOs with $z<0.2$, as described in Section 3. The SEDs are produced using the method described above (including only sources detected in all photometric bands), and the fits are performed using the same models. For a direct comparison, we also carry out the SDSS + UKIDSS+WISE analysis described above, limiting the sources to $z<0.2$. Fit results for both samples are given in Table 2. The SEDs and model fits including GALEX and 2MASS are generally consistent with those of the $z<0.2$ SDSS+UKIDSS+WISE comparison SEDs. The most significant difference compared to the full sample (extending to $z=0.8$ ) is a smaller $F_{\mathrm{AGN}}$ for the quasars at $z<0.2$, as expected for the somewhat lower $L_{[\mathrm{O} \text { III] }}$ values of the low- $z$ sources (discussed further in the next section). Finally, we note that the inclusion of the GALEX data in the QSO 2 SED 
Table 2

Model Fits to Type 1 and 2 QSO Composite SEDs ${ }^{\mathrm{a}}$

\begin{tabular}{|c|c|c|c|c|c|c|c|c|c|c|c|}
\hline Sample & $N_{\mathrm{src}}{ }^{\mathrm{b}}$ & $\langle z\rangle$ & $\left\langle L_{[\mathrm{O}} \mathrm{III]}\right\rangle^{\mathrm{c}}$ & $F_{\mathrm{E}}$ & $F_{\mathrm{Im}}$ & $F_{\mathrm{AGN}}{ }^{\mathrm{d}}$ & $A_{V}$ & $T_{\mathrm{BB}}(\mathrm{K})$ & $\operatorname{Norm}_{B B}{ }^{e}$ & $\log M_{\mathrm{gal}}{ }^{\mathrm{f}}$ & $\log L_{\mathrm{MIR}}^{\mathrm{AGNg}}$ \\
\hline \multicolumn{12}{|c|}{ Full Samples (SDSS+UKIDSS+WISE) } \\
\hline QSO 1 & 334 & 0.45 & 42.6 & $0.42 \pm 0.04$ & $0.02 \pm 0.03$ & $0.56 \pm 0.01$ & 0 & $153 \pm 2$ & $0.038 \pm 0.004$ & $10.88 \pm 0.02$ & $44.85 \pm 0.02$ \\
\hline QSO 2 & 406 & 0.44 & 42.6 & $0.43 \pm 0.03$ & $0.13 \pm 0.01$ & $0.44 \pm 0.01$ & $19 \pm 1$ & $159 \pm 3$ & $0.068 \pm 0.008$ & $10.96 \pm 0.01$ & $44.81 \pm 0.02$ \\
\hline \multicolumn{12}{|c|}{ GALEX+SDSS+2MASS+WISE $(z<0.2)$} \\
\hline QSO 1 & 51 & 0.16 & 42.3 & $0.59 \pm 0.12$ & $0.01 \pm 0.02$ & $0.39 \pm 0.03$ & 0 & $139 \pm 5$ & $0.050 \pm 0.012$ & $10.86 \pm 0.04$ & $44.45 \pm 0.04$ \\
\hline QSO 2 & 76 & 0.15 & 42.3 & $0.54 \pm 0.06$ & $0.12 \pm 0.01$ & $0.34 \pm 0.02$ & $23 \pm 1$ & $145 \pm 2$ & $0.091 \pm 0.007$ & $10.78 \pm 0.02$ & $44.37 \pm 0.03$ \\
\hline \multicolumn{12}{|c|}{ SDSS+UKIDSS+WISE $(z<0.2)$} \\
\hline QSO 1 & 28 & 0.17 & 42.3 & $0.51 \pm 0.18$ & $0.03 \pm 0.03$ & $0.47 \pm 0.05$ & 0 & $142 \pm 4$ & $0.044 \pm 0.008$ & $10.72 \pm 0.05$ & $44.45 \pm 0.06$ \\
\hline QSO 2 & 39 & 0.15 & 42.3 & $0.56 \pm 0.09$ & $0.11 \pm 0.02$ & $0.33 \pm 0.03$ & $22 \pm 2$ & $139 \pm 3$ & $0.121 \pm 0.012$ & $10.75 \pm 0.03$ & $44.31 \pm 0.05$ \\
\hline \multicolumn{12}{|c|}{ [O III] Luminosity Selection $\left.\left(\log L_{[\mathrm{O}} \mathrm{III}\right] /\left[\mathrm{erg} \mathrm{s}^{-1}\right]\right)$} \\
\hline QSO 1 (42.00-42.3) & 67 & 0.31 & 42.1 & $0.53 \pm 0.08$ & $0.03 \pm 0.03$ & $0.44 \pm 0.02$ & 0 & $148 \pm 3$ & $0.039 \pm 0.007$ & $10.78 \pm 0.02$ & $44.50 \pm 0.03$ \\
\hline QSO 1 (42.3-42.5) & 69 & 0.40 & 42.4 & $0.48 \pm 0.06$ & $0.00 \pm 0.01$ & $0.52 \pm 0.02$ & 0 & $152 \pm 2$ & $0.039 \pm 0.004$ & $10.84 \pm 0.03$ & $44.71 \pm 0.03$ \\
\hline QSO $1(42.5-42.7)$ & 89 & 0.47 & 42.6 & $0.37 \pm 0.10$ & $0.00 \pm 0.03$ & $0.63 \pm 0.02$ & 0 & $150 \pm 3$ & $0.056 \pm 0.008$ & $10.79 \pm 0.04$ & $44.88 \pm 0.03$ \\
\hline QSO 1 (42.7-43.0) & 74 & 0.52 & 42.8 & $0.38 \pm 0.09$ & $0.00 \pm 0.06$ & $0.62 \pm 0.02$ & 0 & $152 \pm 3$ & $0.045 \pm 0.006$ & $10.95 \pm 0.03$ & $45.02 \pm 0.03$ \\
\hline QSO 1 (43.0-43.5) & 35 & 0.58 & 43.2 & $0.20 \pm 0.14$ & $0.18 \pm 0.13$ & $0.62 \pm 0.03$ & 0 & $157 \pm 4$ & $0.041 \pm 0.010$ & $11.05 \pm 0.07$ & $45.21 \pm 0.04$ \\
\hline QSO $2(42.00-42.3)$ & 77 & 0.29 & 42.1 & $0.59 \pm 0.07$ & $0.09 \pm 0.02$ & $0.32 \pm 0.02$ & $19 \pm 1$ & $151 \pm 3$ & $0.072 \pm 0.010$ & $10.88 \pm 0.03$ & $44.45 \pm 0.04$ \\
\hline QSO $2(42.3-42.5)$ & 70 & 0.40 & 42.4 & $0.46 \pm 0.08$ & $0.13 \pm 0.03$ & $0.41 \pm 0.03$ & $20 \pm 2$ & $156 \pm 3$ & $0.080 \pm 0.015$ & $10.89 \pm 0.03$ & $44.70 \pm 0.04$ \\
\hline QSO $2(42.5-42.7)$ & 111 & 0.47 & 42.6 & $0.41 \pm 0.04$ & $0.14 \pm 0.02$ & $0.45 \pm 0.01$ & $18 \pm 1$ & $155 \pm 3$ & $0.079 \pm 0.010$ & $10.97 \pm 0.02$ & $44.84 \pm 0.02$ \\
\hline QSO 2 (42.7-43.0) & 103 & 0.51 & 42.8 & $0.35 \pm 0.05$ & $0.16 \pm 0.02$ & $0.49 \pm 0.02$ & $19 \pm 2$ & $162 \pm 3$ & $0.069 \pm 0.010$ & $10.96 \pm 0.02$ & $44.92 \pm 0.03$ \\
\hline QSO 2 (43.0-43.5) & 45 & 0.58 & 43.2 & $0.26 \pm 0.06$ & $0.15 \pm 0.03$ & $0.58 \pm 0.02$ & $17 \pm 2$ & $159 \pm 4$ & $0.082 \pm 0.013$ & $11.11 \pm 0.04$ & $45.23 \pm 0.04$ \\
\hline \multicolumn{12}{|c|}{ W1-W2 Color Selection (Vega) } \\
\hline QSO $1(<0.9)$ & 31 & 0.39 & 42.4 & $0.59 \pm 0.07$ & $0.11 \pm 0.04$ & $0.30 \pm 0.01$ & 0 & $150 \pm 4$ & $0.054 \pm 0.008$ & $10.86 \pm 0.05$ & $44.42 \pm 0.05$ \\
\hline QSO 1 (0.9-1.1) & 179 & 0.41 & 42.5 & $0.47 \pm 0.04$ & $0.03 \pm 0.03$ & $0.50 \pm 0.01$ & 0 & $154 \pm 2$ & $0.034 \pm 0.005$ & $10.88 \pm 0.02$ & $44.74 \pm 0.02$ \\
\hline QSO $1(>1.1)$ & 124 & 0.50 & 42.7 & $0.22 \pm 0.09$ & $0.00 \pm 0.00$ & $0.78 \pm 0.02$ & 0 & $156 \pm 3$ & $0.036 \pm 0.006$ & $10.70 \pm 0.04$ & $45.09 \pm 0.02$ \\
\hline QSO $2(<0.5)$ & 87 & 0.51 & 42.6 & $0.54 \pm 0.06$ & $0.13 \pm 0.01$ & $0.33 \pm 0.02$ & $27 \pm 1$ & $153 \pm 2$ & $0.097 \pm 0.018$ & $11.02 \pm 0.02$ & $44.72 \pm 0.03$ \\
\hline QSO $2(0.5-0.8)$ & 79 & 0.44 & 42.6 & $0.49 \pm 0.05$ & $0.13 \pm 0.02$ & $0.38 \pm 0.02$ & $25 \pm 1$ & $156 \pm 3$ & $0.080 \pm 0.013$ & $10.97 \pm 0.02$ & $44.73 \pm 0.04$ \\
\hline QSO $2(0.8-1.1)$ & 116 & 0.41 & 42.5 & $0.46 \pm 0.04$ & $0.12 \pm 0.01$ & $0.42 \pm 0.01$ & $18 \pm 2$ & $158 \pm 4$ & $0.065 \pm 0.009$ & $10.90 \pm 0.02$ & $44.71 \pm 0.03$ \\
\hline QSO 2 (1.1-1.4) & 91 & 0.44 & 42.7 & $0.33 \pm 0.06$ & $0.11 \pm 0.02$ & $0.56 \pm 0.01$ & $13 \pm 1$ & $155 \pm 4$ & $0.070 \pm 0.013$ & $10.88 \pm 0.03$ & $44.92 \pm 0.03$ \\
\hline QSO $2(>1.4)$ & 33 & 0.41 & 42.7 & $0.14 \pm 0.11$ & $0.08 \pm 0.04$ & $0.78 \pm 0.02$ & $17 \pm 2$ & $149 \pm 7$ & $0.093 \pm 0.024$ & $10.83 \pm 0.05$ & $45.31 \pm 0.04$ \\
\hline \multicolumn{12}{|c|}{ Radio Selection (RL corresponds to $P_{1.4}>10^{24} \mathrm{~W} \mathrm{~Hz}^{-1}$ ) } \\
\hline QSO 1 (RL) & 39 & 0.49 & 42.7 & $0.49 \pm 0.14$ & $0.00 \pm 0.08$ & $0.51 \pm 0.03$ & 0 & $155 \pm 3$ & $0.036 \pm 0.007$ & $11.03 \pm 0.06$ & $44.90 \pm 0.06$ \\
\hline QSO 1 (RQ) & 66 & 0.50 & 42.7 & $0.42 \pm 0.08$ & $0.00 \pm 0.03$ & $0.58 \pm 0.02$ & 0 & $155 \pm 4$ & $0.028 \pm 0.007$ & $10.99 \pm 0.04$ & $44.97 \pm 0.04$ \\
\hline QSO 2 (RL) & 107 & 0.49 & 42.7 & $0.36 \pm 0.07$ & $0.12 \pm 0.02$ & $0.52 \pm 0.02$ & $17 \pm 3$ & $167 \pm 5$ & $0.054 \pm 0.014$ & $11.06 \pm 0.03$ & $45.08 \pm 0.03$ \\
\hline QSO 2 (RQ) & 77 & 0.47 & 42.7 & $0.40 \pm 0.06$ & $0.14 \pm 0.03$ & $0.45 \pm 0.02$ & $18 \pm 2$ & $162 \pm 4$ & $0.063 \pm 0.012$ & $10.94 \pm 0.03$ & $44.84 \pm 0.04$ \\
\hline \multicolumn{12}{|c|}{ Full Samples (SDSS+UKIDSS+WISE); A10 AGN Template } \\
\hline QSO 1 & 334 & 0.45 & 42.6 & $0.65 \pm 0.03$ & $0.09 \pm 0.02$ & $0.26 \pm 0.01$ & 0 & $143 \pm 6$ & $0.017 \pm 0.006$ & $11.12 \pm 0.01$ & $44.84 \pm 0.02$ \\
\hline QSO 2 & 406 & 0.44 & 42.6 & $0.58 \pm 0.03$ & $0.18 \pm 0.01$ & $0.24 \pm 0.01$ & $12 \pm 1$ & $165 \pm 4$ & $0.041 \pm 0.005$ & $10.93 \pm 0.02$ & $44.77 \pm 0.02$ \\
\hline
\end{tabular}

Notes.

The procedure for creating composite SEDs and details of the individual samples are described in Section 4. Uncertainties in all quantities are statistical only and derived from bootstrap resampling.

Number of unique sources included in each composite SED.

Presented in units of $\log \left(L_{[\mathrm{O} \mathrm{III}]} / \mathrm{erg} \mathrm{s}^{-1}\right)$.

The fraction of the total luminosity at $1 \mu \mathrm{m}$ contributed by the AGN, after correcting the AGN component for dust extinction.

e Arbitrary normalization; in each case defined relative to the total SED normalized at $8-13 \mu \mathrm{m}$.

Presented in units of $\log \left(M_{\mathrm{gal}} / M_{\odot}\right)$.

$\mathrm{g}$ The average monochromatic $\nu L_{\nu}$ luminosity at $12 \mu \mathrm{m}$ (in $\log \left(L_{\mathrm{MIR}}^{\mathrm{AGN}} / \mathrm{erg} \mathrm{s}^{-1}\right)$ ) of the quasars in this subsample, assuming a contribution from the AGN and corrected for dust extinction using the best template fit to the composite SED. 


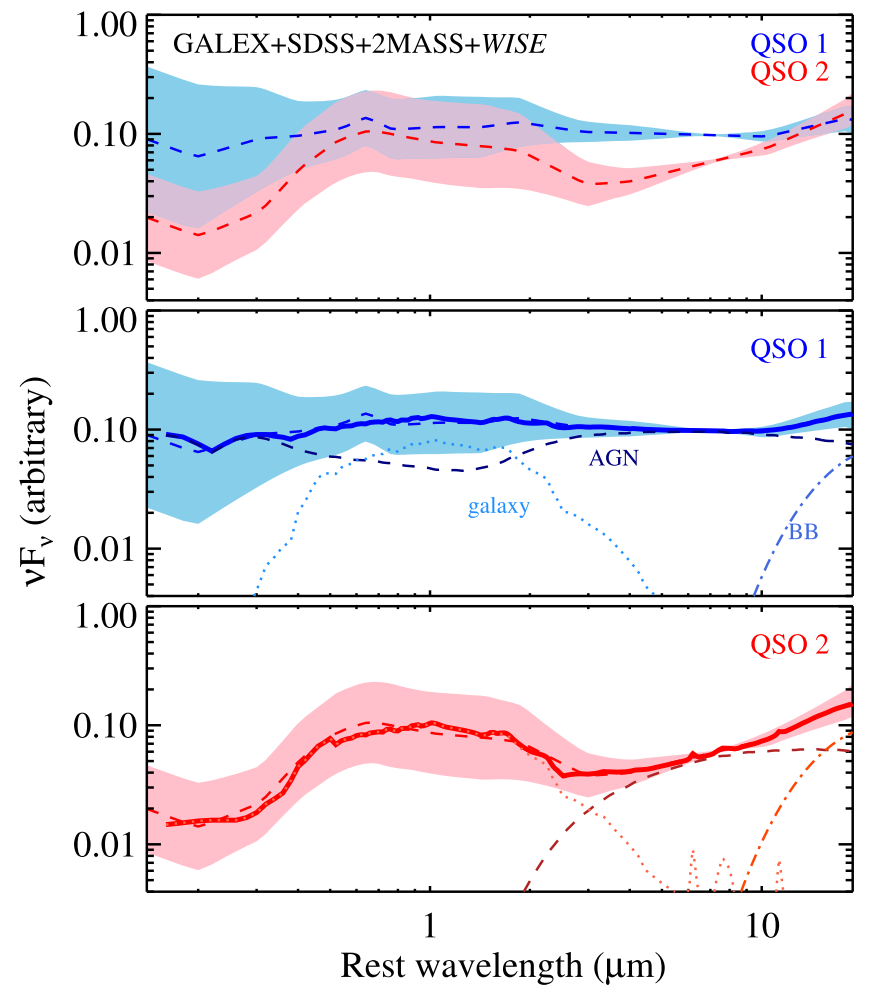

Figure 3. Composite QSO 1 and 2 SEDs and model fits, as shown in Figure 2, but using 2MASS NIR data and UV photometry from GALEX. The quasar samples are limited to sources at $z<0.2$, for which the completeness of 2MASS and GALEX detections is $>93 \%$. These composite SEDs and model fit parameters are similar to those determined using UKIDSS NIR photometry over the redshift range of the full sample, the primary difference being a somewhat smaller contribution from the AGN.

(Figure 3) reveals an upturn at UV wavelengths. This may be due in part to significant star formation in QSO 2 hosts (e.g., Chen et al. 2015), but it may be dominated by scattering from the nuclear source (e.g., Zakamska et al. 2005, 2006; Obied et al. 2016), a component that is not currently included in the SED fits.

\subsection{Dependence of SEDs on $L_{[O ~ I I I}$, MIR Color, and Radio-loudness}

We next examine the average SEDs as a function of quasar physical and observational parameters, specifically $\left.L_{[\mathrm{O}} \mathrm{II}\right]$, WISE color (W1-W2), and radio-loudness. We divide the main (SDSS+UKIDSS+WISE) samples into five bins of $L_{[\mathrm{O} \text { III] }}$ and produce composite SEDs as described in Section 4. The composite SEDs for the highest and lowest $L_{[\mathrm{O} \mathrm{III}]}$ bins are shown in Figure 4. In this figure we normalize the SEDs to have equivalent flux in the total galaxy component at $1 \mu \mathrm{m}$, in order to illustrate the changing contribution of the AGN relative to the galaxy as a function of AGN narrow-line luminosity. In Figure 4(b) we show the ratio of the fluxes of the (unabsorbed) AGN to the total (unabsorbed) SED (AGN and galaxy) at $1 \mu \mathrm{m}\left(F_{\mathrm{AGN}}\right)$. For every bin of $\left.L_{[\mathrm{O}} \mathrm{II}\right], F_{\mathrm{AGN}}$ is higher for the QSO 1s than the QSO 2s, perhaps reflecting the photometric selection of the QSO 1 sample, which biases those objects toward sources in which the active nucleus outshines the galaxy in the optical (e.g., Hopkins et al. 2009). However, for both QSO 1s and QSO 2s, the contribution of the AGN relative to the galaxy increases modestly with increasing $\left.L_{[\mathrm{O}} \mathrm{III}\right]$.
We also compute composite SEDs as a function of W1-W2 color. Short-wavelength MIR color (as with IRAC [3.6] [4.5]) can be used as an AGN indicator and thus a proxy for the dominance of the AGN over the host galaxy (e.g., Stern et al. 2005; Hickox et al. 2007; Stern et al. 2012). We again divide the main (SDSS+UKIDSS+WISE) samples into bins of W1-W2 and produce composite SEDs. The QSO 1s are divided into fewer bins, due to their smaller range in W1-W2 color. The SEDs for the highest and lowest W1-W2 bins, as well as the dependence of $F_{\mathrm{AGN}}$ on $\mathrm{W} 1-\mathrm{W} 2$, are shown in Figure 5. It is immediately clear that W1-W2 color is strongly correlated with $F_{\mathrm{AGN}}$, particularly for QSO 1s and for QSO $2 \mathrm{~s}$ with $\mathrm{W} 1-\mathrm{W} 2>0.8$. For QSO $2 \mathrm{~s}$ with bluer W1-W2, the dependence of $F_{\mathrm{AGN}}$ on $\mathrm{W} 1-\mathrm{W} 2$ is weaker, but there is a stronger correlation between the color and $A_{V}$, as illustrated in Table 2. This suggests that QSO 2s with bluer W1-W2 colors have similar AGN power but higher obscuration, compared to their redder QSO 2 counterparts, as discussed further in Section 5.

As a final comparison, we create composite SEDs for QSO $1 \mathrm{~s}$ and $2 \mathrm{~s}$ divided by radio-loudness between RL and RQ. To specifically check the dependence on radio-loudness, the QSO 1 and QSO 2 RQ samples are further selected to match the $z$ and $\left.L_{[\mathrm{O}} \mathrm{III}\right]$ distributions for the corresponding RL subsets. The composite SEDs are shown in Figure 6, normalized as in Figure 4. It is immediately apparent that the SEDs are comparable, with similar $F_{\mathrm{AGN}}$ values; the trends with $F_{\mathrm{AGN}}$ and radio-loudness are actually opposite for the QSO 1s and QSO 2s. We conclude that there is no strong connection between the optical-MIR SEDs of quasars and the presence or absence of a luminous radio jet.

\subsection{Fitting Results Using A10 AGN Template}

Here we explore the results of our fitting using the AGN template from A10, rather than the template of R06. (We use the primary AGN template provided by A10. That paper also presents an AGN template that is derived starting from the R06 template; the fit results for this template are essentially identical to those described above.) The R06 template was derived from the SEDs of optically identified broad-line quasars, while the A10 template was computed using a combination of unobscured and obscured AGNs identified using a range of methods (X-ray, MIR, and optical) and over a wider range in luminosity. The results of the fits using A10 are shown in Figure 7 and given in Table 2. The model with the A10 AGN template broadly reproduces the shape of the total SEDs, although less well (particularly for QSO 1s) than the fit with the R06 AGN template.

The overall fit results and the comparison between the fit parameters for QSO 1s and 2s are similar between the two AGN templates, with the significant exception being the estimate of $F_{\mathrm{AGN}}$, which is approximately $25 \%$ when using the A10 template, compared to $40 \%-50 \%$ for the R06 template. This difference is due to the fact that the A10 AGN template is weaker in the NIR, so that a stronger galaxy component (corresponding to lower $F_{\mathrm{AGN}}$ ) is needed to fit the observed SEDs (see also A10 for a discussion of this effect). The dependence of $F_{\mathrm{AGN}}$ on $L_{[\mathrm{O} \text { III }]}$ and $\mathrm{W} 1-\mathrm{W} 2$ is similar between the two templates, with the A10 template fitting showing better agreement in $F_{\mathrm{AGN}}$ between the two QSO types. The other clear difference between the results is that the A10 template is redder in the MIR, requiring a somewhat weaker blackbody 

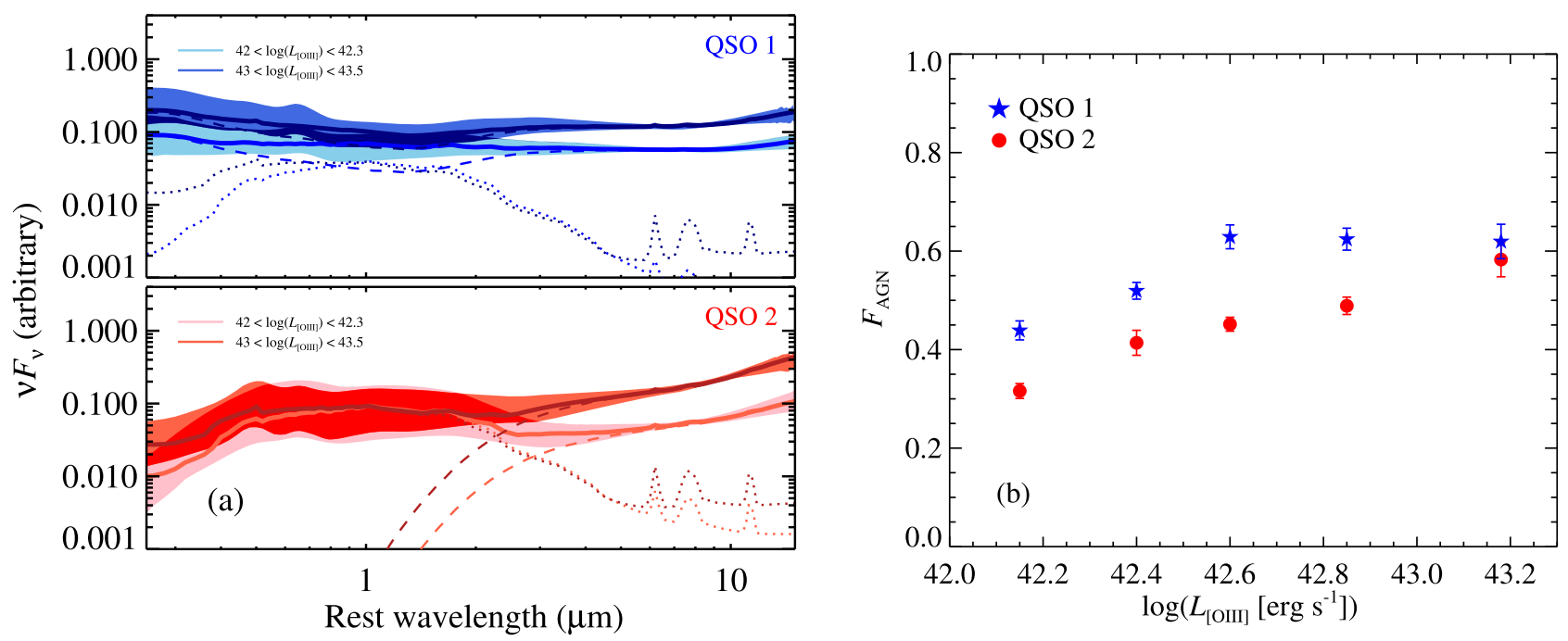

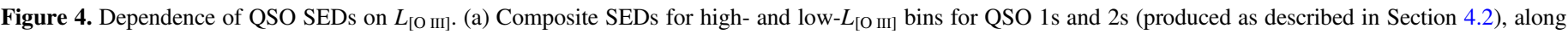

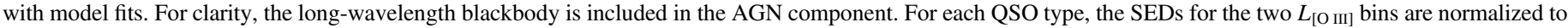

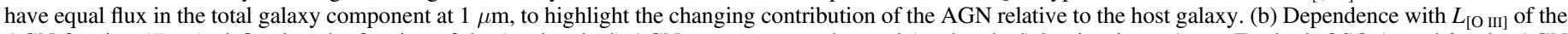

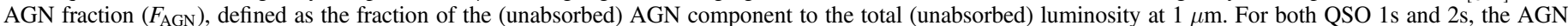
contributes a relatively larger fraction to the total luminosity as $L_{[\mathrm{O} \text { III] }}$ increases.

component relative to the fits with the R06 AGN template. We note, however, that for both sets of templates the QSO $2 \mathrm{~s}$ require a stronger blackbody component compared to the QSO $1 \mathrm{~s}$, reflecting the different shapes of the two SEDs. Using the A10 template, the fits are consistent between RL and RQ QSOs, similar to what is found with the R06 template.

We note that the composite SEDs presented here are created using broadly similar techniques to those in R06 and A10, so the reasons for the differences between the templates are not immediately clear. The A10 template was constructed from AGNs extending to lower luminosities than those for R06. The fact that our composite SEDs for QSOs are best fitted by the R06 template may imply a dependence of the intrinsic AGN SED with luminosity. The A10 procedure also included a more self-consistent treatment of the host galaxy emission, and A10 suggests that the R06 template may be brighter in the NIR owing to residual host galaxy contamination. In contrast, it may be possible that the redder colors of the A10 template at long wavelengths are due to some cooler dust emission that either is characteristic of lower-luminosity AGNs or originates in starforming regions in the host galaxy. (The need for a stronger long-wavelength blackbody component in our QSO 2s suggests that this emission may be connected to AGN obscuration.) Overall, we conclude that the choice of AGN template has some impact on the ultimate fit parameters, so that it is difficult to draw strong conclusions about the absolute strength of the AGN relative to the galaxy luminosity. However, our primary conclusions about the relative shapes of the QSO 1 and 2 SEDs and their dependence on luminosity, MIR color, and radioloudness are independent of the AGN template used.

\section{Photometric Selection of Luminous Obscured Quasars}

A primary challenge for studying the large populations of obscured quasars detected with WISE is selecting these objects based on optical and MIR photometric data alone, in the absence of spectroscopic or other multiwavelength indicators. In this section we examine the observed colors of the SDSS QSO 1 and 2 populations and explore how the observed colors of the model SEDs in Section 4 vary with redshift. Here we focus on colors from WISE and SDSS, which are among the largest-area MIR and optical surveys with the depth required to detect obscured quasars to $z>1$ (e.g., Assef et al. 2013; DiPompeo et al. 2014, 2015).

\subsection{Quasar Selection Using Mid-infrared Colors}

A number of simple criteria for selecting AGNs using MIR data from WISE have been proposed (e.g., Jarrett et al. 2011; Mateos et al. 2012; Stern et al. 2012), following on similar efforts using Spitzer IRAC and MIPS data (Lacy et al. 2004; Rowan-Robinson et al. 2005; Stern et al. 2005; Donley et al. 2012). Of the WISE criteria, the simplest is that proposed by Stern et al. (2012), consisting of a single cut in the W1-W2 color with W1-W2 $>0.8$ (Vega), for sources with a flux threshold of $\mathrm{W} 2<15.05$. This cut picks out objects with red NIR SEDs and thus separates the (red) hot dust continuum from AGNs from the (blue) approximately Rayleigh-Jeans tail of the stellar continuum, and is roughly equivalent to the lower bound of the IRAC "wedge" selection presented in Stern et al. (2005). To probe to fainter WISE fluxes, Assef et al. (2013) proposed a W1-W2 threshold that is dependent on W2 magnitude (with the threshold becoming redder for fainter W2 sources) that achieves $\sim 90 \%$ reliability for $\mathrm{W} 2<17.11$. Other studies have proposed additional cuts making use of the $12 \mu \mathrm{m}$ band, specifically the W2-W3 color (Jarrett et al. 2011; Mateos et al. 2012), to more efficiently remove contamination from star-forming galaxies. The $22 \mu \mathrm{m}$ WISE band is generally not useful for AGN selection at medium to high redshift, because of its significantly poorer sensitivity relative to the three bluer bands (e.g., Mateos et al. 2012).

To compare to these selection criteria, in Figure 8(a) we show the observed W1-W2 and W2-W3 colors for the SDSS QSO 1s and QSO 2s, along with the distribution for WISE sources with W3 detections and W2 $<15.05$ (Vega), as selected in recent studies of WISE quasars (DiPompeo et al. 2014, 2015). Almost all (98\%) of the QSO 1s and roughly half (49\%) of QSO 2s have red W1-W2 colors that would be 

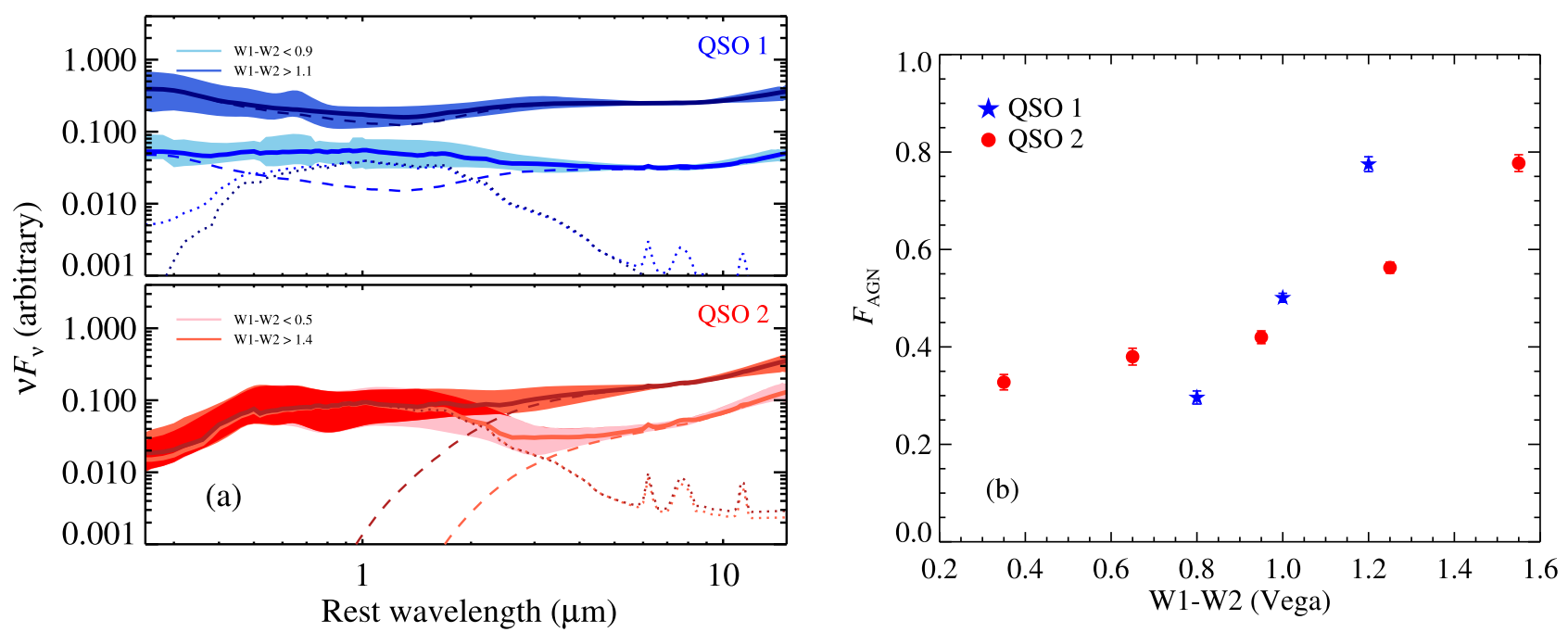

Figure 5. Dependence of QSO SEDs on observed W1-W2. (a) Composite SEDs for sources with blue and red colors for QSO 1s and 2s (produced as described in Section 4.2), along with model fits, and normalized as in Figure 4. (b) Dependence of $F_{\mathrm{AGN}}$ with observed W1-W2 color. For both QSO 1s and 2s, the AGN contributes a relatively larger fraction to the total luminosity for redder W1-W2, but the dependence is much steeper for QSO 1s, which occupy a smaller overall range in $\mathrm{W} 1-\mathrm{W} 2$.

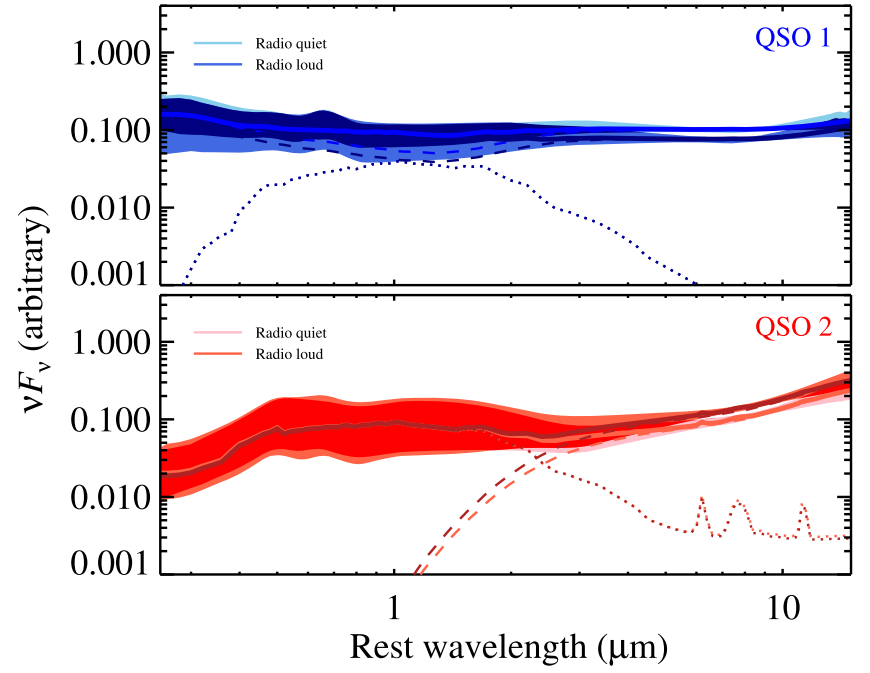

Figure 6. Composite SEDs, as shown in Figure 4, for the QSOs divided into RL and RQ subsets based on a $1.4 \mathrm{GHz}$ monochromatic luminosity threshold of $P_{1.4}>10^{24} \mathrm{~W} \mathrm{~Hz}^{-1}$. The composite SEDs of both types of quasar show only a weak dependence of the SEDs on radio-loudness.

selected by a cut of $\mathrm{W} 1-\mathrm{W} 2>0.7$ (Vega). In comparison, $18 \%$ of the general-population bright WISE sources would be selected using this cut. The QSO 2s have a wider range of $\mathrm{W} 1-\mathrm{W} 2$ colors and are markedly redder in W2-W3, reflecting the redder MIR continuum that is apparent in the composite SEDs (Figure 2). This suggests the possibility that obscured quasars might be selected on the basis of their MIR colors alone, with a nominal division around $\mathrm{W} 2-\mathrm{W} 3=3.3$. However, it is immediately clear from Figure 8(a) that this selection would retain some contamination from unobscured quasars.

We further explore how the observed colors of QSO 1s and $2 \mathrm{~s}$ will change with redshift, making use of the model fits to the composite QSO 1 and 2 SEDs presented in Section 4. We calculate the observed colors of these SEDs as a function of redshift and compare the colors of the two types of quasars.

In this exercise, we do not simply use the best-fit models for the composite SEDs at all redshifts. This is because, as a flux- limited data set, the AllWISE catalog is sensitive to sources with higher luminosities at high redshift. In particular, we will be limited by the observed MIR luminosity, which is dominated by the AGN component, and so more luminous AGNs will be selected at higher redshift. In contrast, we may expect the overall host galaxy luminosity to change more slowly with redshift, as quasars are hosted in systems of similar mass at all redshifts (e.g., Myers et al. 2007; Ross et al. 2009; Hickox et al. 2011; DiPompeo et al. 2016, 2017). Thus, at higher redshift, the WISE catalog will preferentially include quasars with a higher fraction of AGNs to host galaxy luminosity; we have confirmed this by fits to composite QSO SEDs in bins of redshift, showing that $F_{\mathrm{AGN}}$ rises from $\approx 0.4$ at $z=0.3$ to $\approx 0.6$ at $z=0.8$. In producing our model curves, we use our best-fit components for the QSO 1s and 2s but vary the normalization of the AGN components relative to the host galaxy, so that it follows an extrapolation of the trend observed at lower redshift and reaches $F_{\mathrm{AGN}} \approx 0.8$ at $z=2$. For comparison, we also compute the equivalent model curves for the fits using the A10 AGN template, with the corresponding variation in $F_{\mathrm{AGN}}$ with redshift.

The observed colors are derived by convolving the model SEDs with the response functions of the SDSS and WISE filters. The resulting $\mathrm{W} 1-\mathrm{W} 2$ and $\mathrm{W} 2-\mathrm{W} 3$ colors for the redshift range $0<z<2$ are shown by the tracks in Figure 8(a). (Given the WISE $12 \mu \mathrm{m}$ flux limit, we expect to detect relatively few obscured quasars at $z>2$.) The tracks using the R06 and A10 templates are shown as solid and dotted lines, respectively. Both composite quasar SEDs have red W1-W2 colors at all redshifts, but we note that many obscured quasars are significantly redder in $\mathrm{W} 2-\mathrm{W} 3$, and many are bluer in W1-W2, than most of the X-ray AGNs used in some previous studies of WISE color selection (e.g., Mateos et al. 2012; Stern et al. 2012). This may be due to the fact that blue W1-W2 colors are associated with higher levels of obscuration that could absorb X-ray emission strongly enough to drop below survey flux limits (e.g., Lansbury et al. 2014, 2015; Stern et al. 2014). Follow-up spectroscopic and X-ray observations of sources toward the bottom right of WISE color-color space indeed confirm their AGN nature (Hainline 

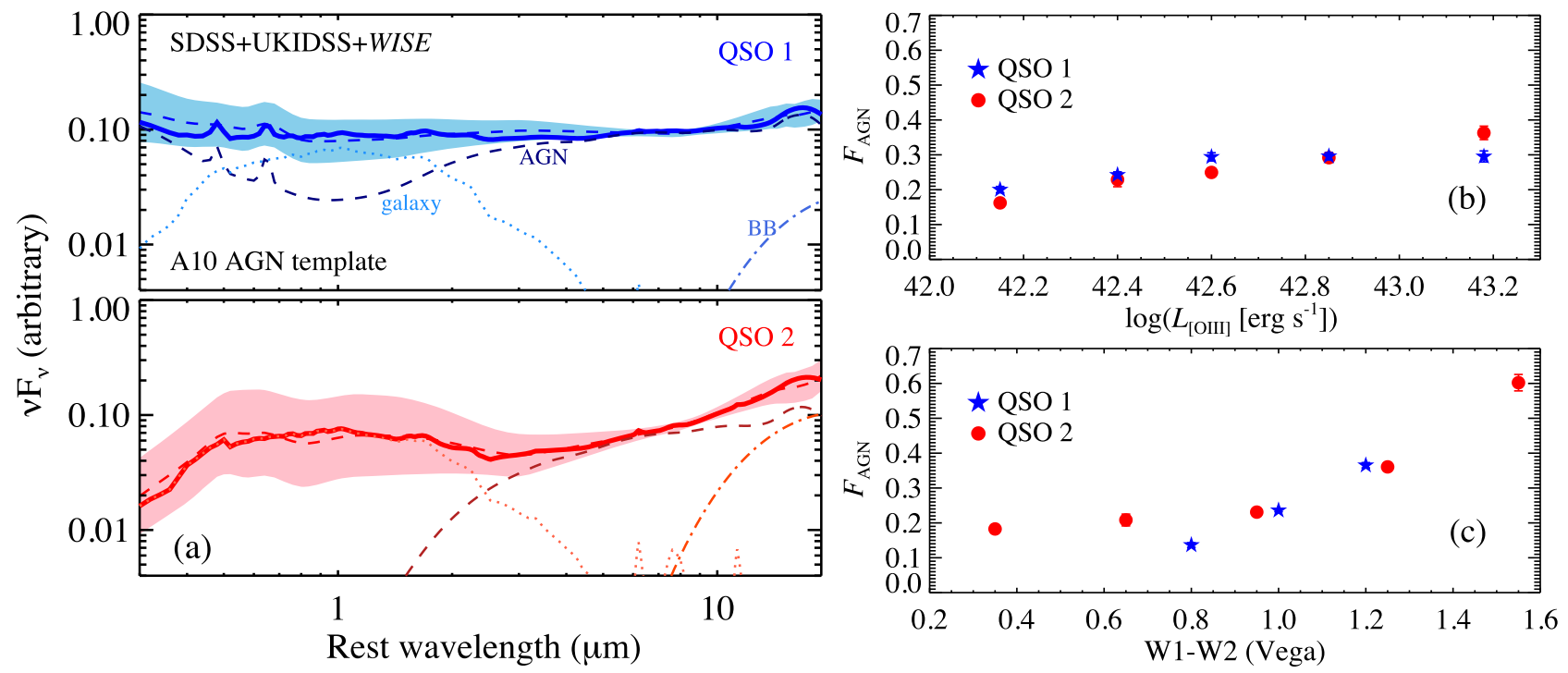

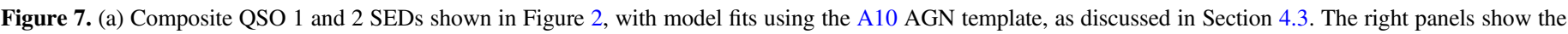

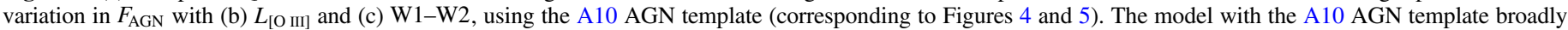

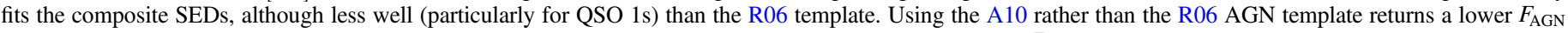
with better correspondence between QSO 1s and 2s; the two AGN templates yield similar trends between $F_{\mathrm{AGN}}, L_{[0 \mathrm{III}]}$, and W1-W2.

et al. 2014a; Hviding et al. 2017), as well as heavy X-ray obscuration (W. Yan et al. 2017, in preparation).

\subsection{Distinguishing between Obscured and Unobscured Quasars}

We have thus established that obscured and unobscured quasars have characteristic colors in the MIR that can be used to distinguish them from galaxies, and that this selection is able to identify almost all QSO $1 \mathrm{~s}$ and a substantial fraction of QSO $2 \mathrm{~s}$. Here we explore the possibility of distinguishing between the two types of quasars using further color criteria.

As clearly shown in Figure 8(a), there is significant separation between QSO $1 \mathrm{~s}$ and $2 \mathrm{~s}$ in the WISE colors. At low redshift, the QSO 2 SED is redder than the QSO 1 SED in W2-W3, raising the possibility of using WISE photometry alone to both select quasars and distinguish between obscured and unobscured subsets. However, there is overlap in W2-W3 between the high- $z$ QSO $2 \mathrm{~s}$ and the QSO 1s, meaning that without further redshift information it is difficult to define a single W2-W3 color cut that efficiently separates QSO 1s and 2 s.

The top panel of Figure 9 shows the redshift tracks in W2-W3 for the QSO 1 and 2 SEDs. (Model curves for the A10 template are plotted as dotted lines, showing similar trends.) For the QSO $2 \mathrm{~s}$, we explore the effects of obscuration by including models with $A_{V}=4$ and 40 , as well as $A_{V}=20$, which best fits the composite SED. For W2-W3, there is clear overlap between high- $z$ QSO 1s and low- $z$ QSO $2 \mathrm{~s}$, and the color depends significantly on $A_{V}$. This suggests that W2-W3 may have limited utility for separating the two quasar types; however, choosing only objects that are very blue (W2-W3 $<2.5$ ) or very red (W2-W3 $>4$ ) is likely to yield relatively clean, if incomplete, samples of obscured and unobscured quasars.

A more effective method to separate obscured and unobscured quasars is based on their observed optical-MIR color (e.g., Hickox et al. 2007; DiPompeo et al. 2015, 2016, 2017; Donoso et al. 2014; Chen et al. 2015). By definition, obscured quasars are extinguished in the optical and UV and thus are dominated by their host galaxies in those bands, while the MIR is relatively unaffected by obscuration. For the most effective selection, we first examine the $u-\mathrm{W} 3$ color, which provides the widest baseline between SDSS and WISE photometric bands (excluding the $22 \mu \mathrm{m}$ band, due to its poorer sensitivity). The observed $u-\mathrm{W} 3$ color and the tracks for the composite SEDs are shown in Figure 8(b) and the middle panel of Figure 9. These figures show a clear separation between the observed colors of QSO 1s and 2s, and the two model SEDs have significantly different colors for all redshifts and $A_{V}$. Thus, for $u$ and $12 \mu \mathrm{m}$ detections (or $u$-band upper limits), this provides a very effective method for distinguishing between obscured and unobscured quasars. We define a selection criterion, shown by the dashed line in Figure 8(b), that optimizes the separation between QSO 1s and $2 \mathrm{~s}$ that can also be selected with $\mathrm{W} 1-\mathrm{W} 2>0.7$. This is given by $(u-\mathrm{W} 3[\mathrm{AB}])>1.4(\mathrm{~W} 1-\mathrm{W} 2$ [Vega] $)+3.2$. This criterion identifies $93 \%$ of the QSO 1s and $92 \%$ of the QSO 2s in our sample $(94 \%$ and $95 \%$, respectively, of those with $\mathrm{W} 1-$ $\mathrm{W} 2>0.7)$.

It is also useful to explore criteria that can select obscured quasars in bands that are more sensitive than the SDSS $u$ and WISE $12 \mu \mathrm{m}$ bands. One such criterion was proposed by Hickox et al. (2007) using IRAC data, for which a cut of $R-[4.5]>3.1$ (AB) provided clean separation between IRselected obscured and unobscured quasars at $z>0.7$; this criterion has since been used by a number of studies with optical and WISE data (e.g., DiPompeo et al. 2014, 2015, 2016, 2017; Donoso et al. 2014; Mendez et al. 2016). Tracks for the corresponding SDSS and WISE color $(r-\mathrm{W} 2)$ for the model SEDs are shown in the bottom panel of Figure 9. These observed colors are not able to cleanly separate QSO 1s and $2 \mathrm{~s}$ at low redshift (as also observed with Spitzer data by Hickox et al. 2007), but the separation becomes most significant at $z>0.5$ as the $4000 \AA$ break moves into the $r$ band, making this a particularly useful criterion for selecting obscured quasars if there is some redshift information. (We note that, in principle, with estimates of redshift one can 

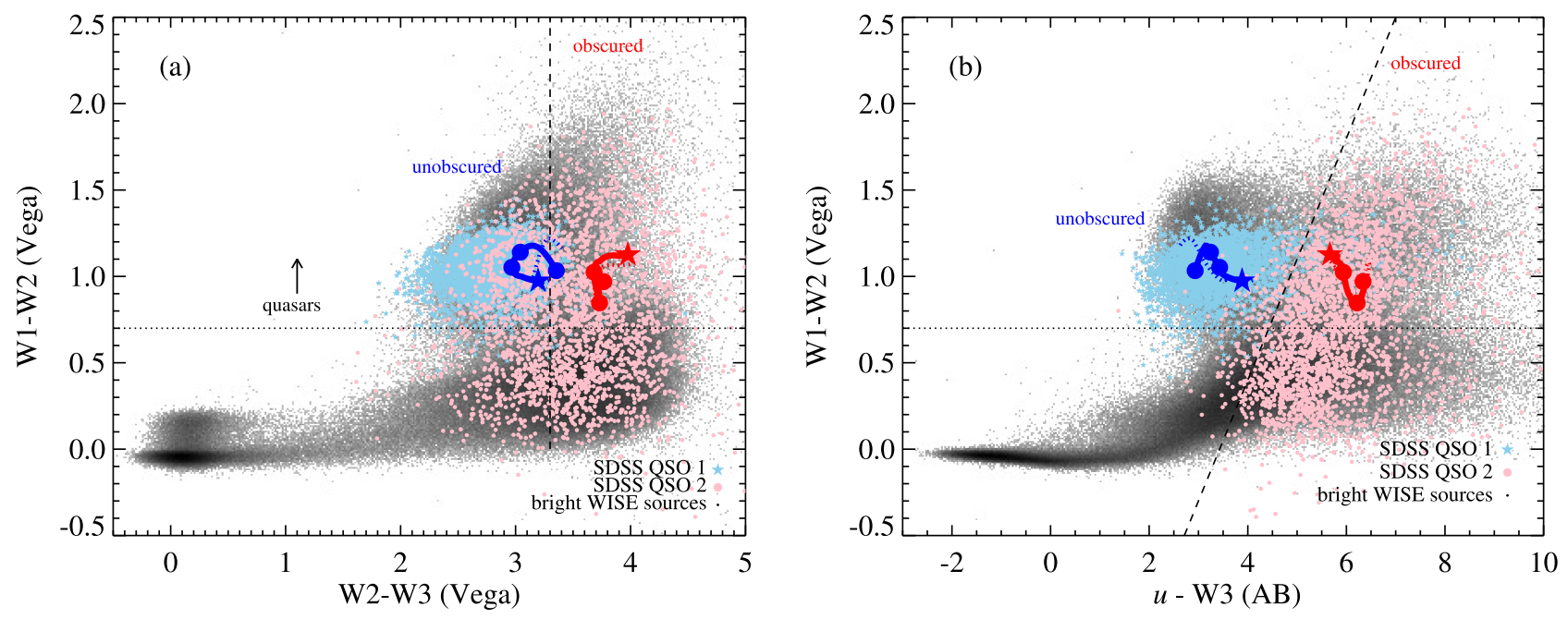

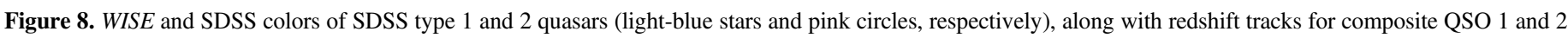

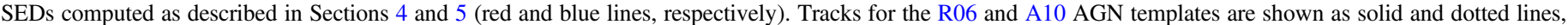

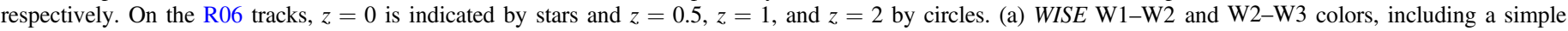

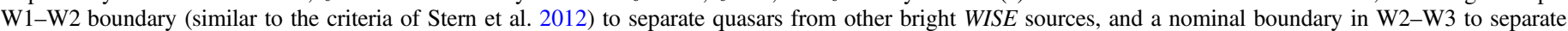

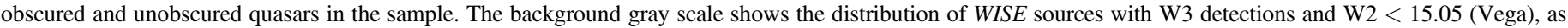

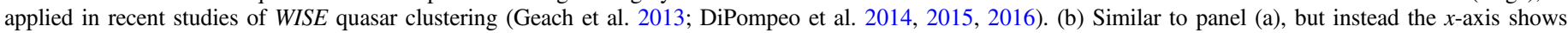

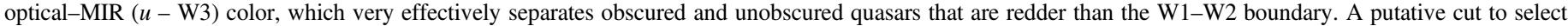

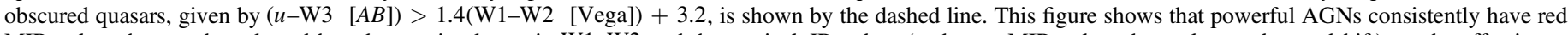

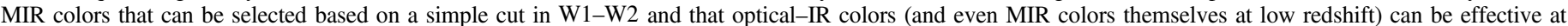

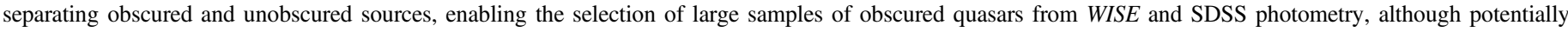
missing many heavily obscured sources with red W1-W2 colors.

identify the level of quasar obscuration based on full model fits to the SEDs, for example, as performed in Hainline et al. (2014a) and Hviding et al. (2017). However, in some cases only uncertain photometric redshifts are available, and full SED fitting can be computationally expensive for large samples, so that there can be significant utility in simple photometric color selection.)

We conclude that optical-IR color selection criteria can be effective at distinguishing between obscured and unobscured quasars, providing a tool to select large numbers of obscured quasars selected based solely on WISE and optical photometry.

\section{Discussion}

The primary conclusion that emerges from this work is the intrinsic similarity of the optical-MIR SEDs of obscured and unobscured quasars, for which the only marked difference in the SED shape appears to be due to reddening of the AGN component. Further, we find no substantial difference in composite SEDs as a function of radio-loudness, confirming the broad conclusions of previous SED analyses for Type 1 quasars (e.g., Elvis et al. 1994). In contrast, there is significant dependence of the observed SED on intrinsic AGN luminosity (parameterized here by $L_{[\mathrm{O}}{ }_{\mathrm{III}}$ ) as the radiative AGN emission becomes more prominent relative to the host galaxy. This contrast suggests a weak connection between the mechanical power of the quasar (as measured by radio luminosity) and the radiative power, with implications for the launching mechanisms of relativistic jets (e.g., Sikora et al. 2007; Tadhunter 2016; Padovani et al. 2017).

The overall uniformity of intrinsic quasar SEDs confirms that the population of luminous AGNs may be modeled using relatively simple prescriptions that are independent of obscuration of the central engine or the presence of relativistic jets. We therefore have a robust understanding of photometric selection of obscured quasars based on optical and MIR data and verify simple color criteria that have been used in previous work (e.g., Hickox et al. 2007, 2011; DiPompeo et al. 2014, 2015, 2016; Donoso et al. 2014).

We emphasize, however, that obscured quasar color selection is dependent on redshift, illustrating the need in pure photometric selection for redshift estimates (e.g., utilizing galactic features in the rest-frame optical spectrum) that can be obtained using template fitting (e.g., Hickox et al. 2007; Chung et al. 2014; Hainline et al. 2014a) or machine-learning techniques (e.g., Brodwin et al. 2006; Geach 2012). We also note that simple MIR color techniques are likely to miss the most heavily obscured AGNs, which have MIR colors consistent with star-forming galaxies at similar redshift (e.g., Hainline et al. 2016b; Yuan et al. 2016).

A related point is that the observed SEDs of type 1 and 2 quasars differ even well into the rest-frame MIR, indicating that dust affects even the longest wavelengths probed by WISE. This highlights the challenge of fully correcting for these effects and selecting samples of obscured and unobscured quasars matched in intrinsic luminosity based on photometry alone (e.g., Hickox et al. 2007). The extinction of the AGN component in the observed WISE bands may also contribute to the larger flux of the long-wavelength blackbody component that we obtain for the QSO 2s compared to the QSO 1s; a more complete correction for this extinction could, in principle, yield more consistent contributions of the AGN relative to the galaxy in the two types of QSOs. Alternatively, the stronger longwavelength emission in QSO 2s could be due to cooler dust heated by star formation, which may be connected to obscuration in quasars (e.g., Page et al. 2004; Chen et al. 2015). Finally, we note that the observed SED differences raise the possibility that quasar MIR SEDs and inferred intrinsic luminosities are influenced by orientation effects owing to anisotropic absorption and emission (e.g., Hönig 


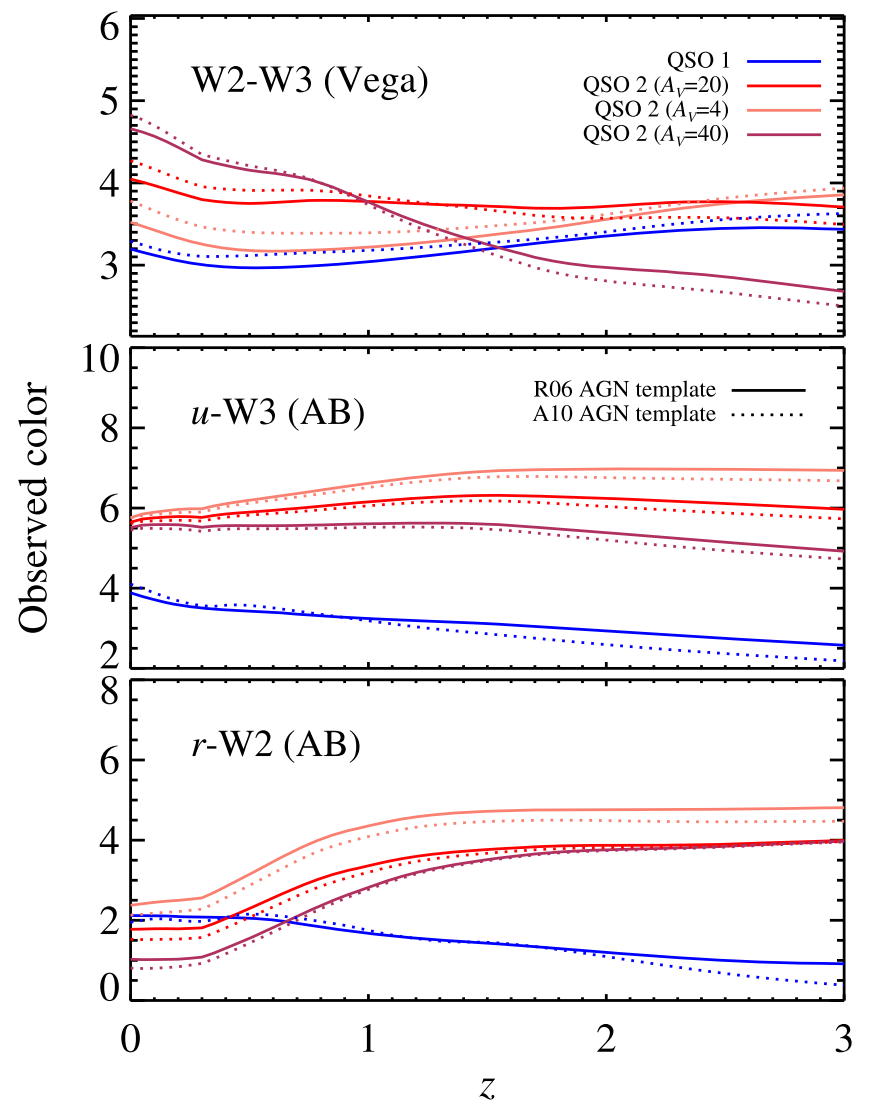

Figure 9. Model tracks for MIR and optical-IR colors using the QSO 1 and 2 composite SED fits, as described in Section 5. Tracks are shown for three different levels of obscuration in the QSO $2 \mathrm{~s}\left(A_{V}=4,20\right.$, and 40). The top panel shows that W2-W3 color can distinguish between obscured and unobscured quasars, but only at low redshift. The middle and bottom panels show that optical-IR colors are effective at separating the two types of quasars, with clear separation in $u-\mathrm{W} 3$ at all redshifts, while $r-\mathrm{W} 2$ distinguishes between them at $z>0.5$.

et al. 2011; Podigachoski et al. 2015). This anisotropy is a natural product of some models of a circumnuclear torus (e.g., Nenkova et al. 2008; Netzer 2015; Stalevski et al. 2016), while the simple extinction prescription adopted here is more appropriate for a foreground "screen" of dust on larger scales in the host galaxy (e.g., Goulding et al. 2012; Chen et al. 2015; Glikman et al. 2015).

Based on the SED fits, we can estimate the stellar masses corresponding to the "average" host galaxy components. We use the average total luminosities of the quasar in each sample at rest-frame $K$ band $(2.1 \mu \mathrm{m})$ and compute the average galaxy luminosity from the components of the best-fit SED model. We then convert to an approximate galaxy stellar mass assuming a $K$-band mass-to-light ratio of 3.1 (Courteau et al. 2014). The resulting galaxy masses are listed in Table 2 . For reference, we similarly compute the unabsorbed rest-frame $12 \mu \mathrm{m}$ luminosity $L_{\mathrm{MIR}}^{\mathrm{AGN}}$ for each quasar subset, also listed in Table 2. For each quantity, the statistical uncertainties are derived from bootstrap resampling.

The average QSO 1 and 2 SEDs correspond to similar galaxy stellar masses of $\log M_{\mathrm{gal}} / M_{\odot} \approx 11$. The precise masses depend on the choice of AGN template and so are difficult to estimate precisely; for the R06 AGN template, the resulting QSO 2 galaxy masses are modestly larger than those for the QSO 1s, consistent with the latest measurements of WISE-selected quasar clustering (e.g., DiPompeo et al. 2016, 2017) that indicate that QSO 2s lie in more massive systems. However, for the A10 template, the QSO 1s are correspondingly more massive, in conflict with the simplest interpretation of the clustering results. These results highlight the challenges in characterizing absolute galaxy masses for AGN hosts from analyses of broadband SEDs and motivate continued study of the shape of the intrinsic AGN SED and its variation with AGN properties.

Independent of the uncertainty on the stellar masses, the dependence of the SED shape for each type of QSO on luminosity (Figure 4) tells us something about the Eddington ratio distribution for luminous quasars. If the luminosity and thus mass of a galaxy correlate broadly with $\mathrm{BH}$ mass (due to $\mathrm{BH}-$ galaxy correlations; for a review see Kormendy \& Ho 2013), then we can interpret $F_{\mathrm{AGN}}$ as a rough proxy for $L_{\mathrm{AGN}} / M_{\mathrm{BH}}$ and thus the Eddington ratio. We find that $F_{\mathrm{AGN}}$ increases with $L_{[\mathrm{O} \text { III] }}$, but only modestly (a factor of 10 in $L_{[\mathrm{O} \text { III] }}$ corresponds to a factor of $\sim 2$ in $F_{\mathrm{AGN}}$ ). This therefore indicates that quasars of higher luminosity have higher $\mathrm{BH}$ masses and higher Eddington ratios, consistent with a scenario in which AGNs with a given BH mass occupy a wide range in accretion rates (e.g., Hopkins \& Hernquist 2009; Aird et al. 2012; Hickox et al. 2014; Jones et al. 2016, 2017).

Finally, we use our proposed selection cuts to estimate the size of the population of quasars that can be identified using WISE photometry, based on our previous studies of WISE quasars (e.g., Geach et al. 2013; DiPompeo et al. 2014, 2015) and the well-studied IRAC-selected quasar sample of Hickox et al. (2007) in the Boötes field. Adopting the color criteria of $\mathrm{W} 1-\mathrm{W} 2>0.8$ (Vega) and W2 $<15.05$ (as per Stern et al. 2012) and $L_{\text {bol }} \gtrsim 10^{45} \mathrm{erg} \mathrm{s}^{-1}$ ("quasar" luminosities), we obtain a population of $\sim 40$ unobscured and $\sim 20$ obscured quasars per $\mathrm{deg}^{2}$, with a significant tail out to $z \gtrsim 2$. (While the observed obscured population is smaller, previous studies suggest that after accounting for obscuration and flux limits the intrinsic populations of obscured and unobscured IR-selected quasars are close to equal in number; e.g., Hickox et al. 2007; Assef et al. 2015.) Over the whole sky, this corresponds to $\sim 2.5$ million WISE-selected quasars, with $\sim 900,000$ sources that would be selected as obscured based on a color criterion of $r-\mathrm{W} 2>3.1$ (AB), as employed in some previous work (DiPompeo et al. 2014, 2015, 2016, 2017). These estimates highlight the power of WISE and SDSS photometric selection in identifying large samples of obscured quasars that are dramatically expanding our understanding of the population of rapidly growing $\mathrm{BHs}$.

R.C.H. acknowledges support from the National Science Foundation through AST grant Nos. 1211112 and 1515404 and CAREER grant 1554584, from NASA through grants NNX16AN48G and NNX15AU32H, and from an Alfred P. Sloan Research Fellowship. A.D.M. acknowledges support from the National Science Foundation through AST grant No. 1515404. R.C.H., A.D.M., K.N.H., and M.A.D. acknowledge support from NASA for ADAP grant Nos. NNX12AE38G and NNX15AP24G.

This publication makes use of data products from the Widefield Infrared Survey Explorer, which is a joint project of the University of California, Los Angeles, and the Jet Propulsion Laboratory/California Institute of Technology, funded by the National Aeronautics and Space Administration. This work is 
based in part on data obtained as part of the UKIRT Infrared Deep Sky Survey.

This publication makes use of data products from the Two Micron All Sky Survey, which is a joint project of the University of Massachusetts and the Infrared Processing and Analysis Center/California Institute of Technology, funded by the National Aeronautics and Space Administration and the National Science Foundation.

Funding for SDSS-III has been provided by the Alfred P. Sloan Foundation, the Participating Institutions, the National Science Foundation, and the U.S. Department of Energy Office of Science. The SDSS-III Web site is http://www.sdss3.org/.

SDSS-III is managed by the Astrophysical Research Consortium for the Participating Institutions of the SDSS-III Collaboration, including the University of Arizona, the Brazilian Participation Group, Brookhaven National Laboratory, Carnegie Mellon University, University of Florida, the French Participation Group, the German Participation Group, Harvard University, the Instituto de Astrofisica de Canarias, the Michigan State/Notre Dame/JINA Participation Group, Johns Hopkins University, Lawrence Berkeley National Laboratory, Max Planck Institute for Astrophysics, Max Planck Institute for Extraterrestrial Physics, New Mexico State University, New York University, Ohio State University, Pennsylvania State University, University of Portsmouth, Princeton University, the Spanish Participation Group, University of Tokyo, University of Utah, Vanderbilt University, University of Virginia, University of Washington, and Yale University.

\section{ORCID iDs}

Ryan C. Hickox (iD https://orcid.org/0000-0003-1468-9526

Kevin N. Hainline (D) https://orcid.org/0000-0003-4565-8239

Nadia L. Zakamska (i) https://orcid.org/0000-0001-6100-6869

\section{References}

Abazajian, K. N., Adelman-McCarthy, J. K., Agüeros, M. A., et al. 2009, ApJS, 182, 543

Ahn, C. P., Alexandroff, R., Allende Prieto, C., et al. 2012, ApJS, 203, 21

Aird, J., Coil, A. L., Moustakas, J., et al. 2012, ApJ, 746, 90

Alexander, D. M., Brandt, W. N., Hornschemeier, A. E., et al. 2001, AJ, 122,2156

Alexander, D. M., \& Hickox, R. C. 2012, NewAR, 56, 93

Alonso-Herrero, A., Pérez-González, P. G., Alexander, D. M., et al. 2006, ApJ, 640, 167

Antonucci, R. 1993, ARA\&A, 31, 473

Assef, R. J., Eisenhardt, P. R. M., Stern, D., et al. 2015, ApJ, 804, 27

Assef, R. J., Kochanek, C. S., Brodwin, M., et al. 2010, ApJ, 713, 970

Assef, R. J., Stern, D., Noirot, G., et al. 2017, ApJS, submitted (arXiv:1706. 09901)

Assef, R. J., Stern, D., Kochanek, C. S., et al. 2013, ApJ, 772, 26

Baldwin, J. A., Phillips, M. M., \& Terlevich, R. 1981, PASP, 93, 5

Berney, S., Koss, M., Trakhtenbrot, B., et al. 2015, MNRAS, 454, 3622

Bianchi, L. 2011, Ap\&SS, 335, 51

Bianchi, L. 2014, Ap\&SS, 354, 103

Bianchi, L., Herald, J., Efremova, B., et al. 2011, Ap\&SS, 335, 161

Brodwin, M., Brown, M. J. I., Ashby, M. L. N., et al. 2006, ApJ, 651, 791

Brown, M. J. I., et al. 2008, ApJ, 682, 937

Chen, C.-T. J., Hickox, R. C., Alberts, S., et al. 2015, ApJ, 802, 50

Chung, S. M., Kochanek, C. S., Assef, R., et al. 2014, ApJ, 790, 54

Cohen, M., Wheaton, W. A., \& Megeath, S. T. 2003, AJ, 126, 1090

Coil, A. L., Hennawi, J. F., Newman, J. A., Cooper, M. C., \& Davis, M. 2007, ApJ, 654, 115

Coil, A. L., Newman, J. A., Croton, D., et al. 2008, ApJ, 672, 153

Comastri, A., Setti, G., Zamorani, G., \& Hasinger, G. 1995, A\&A, 296, 1

Condon, J. J., Cotton, W. D., Greisen, E. W., et al. 1998, AJ, 115, 1693

Conroy, C., \& White, M. 2013, ApJ, 762, 70
Courteau, S., Cappellari, M., de Jong, R. S., et al. 2014, RvMP, 86, 47 Croom, S. M., Boyle, B. J., Shanks, T., et al. 2005, MNRAS, 356, 415 Croom, S. M., Smith, R. J., Boyle, B. J., et al. 2004, MNRAS, 349, 1397 Croton, D. J. 2009, MNRAS, 394, 1109

Cutri, R. M., Skrutskie, M. F., van Dyk, S., et al. 2003, VizieR Online Data Catalog: 2MASS All-Sky Catalog of Point Sources, 2246

da Ângela, J., Shanks, T., Croom, S. M., et al. 2008, MNRAS, 383, 565

Dai, Y. S., Elvis, M., Bergeron, J., et al. 2014, ApJ, 791, 113

Del Moro, A., Mullaney, J. R., Alexander, D. M., et al. 2014, ApJ, 786, 16

Di Matteo, T., Springel, V., \& Hernquist, L. 2005, Natur, 433, 604

DiPompeo, M. A., Hickox, R. C., Eftekharzadeh, S., \& Myers, A. D. 2017, MNRAS, 469, 4630

DiPompeo, M. A., Hickox, R. C., \& Myers, A. D. 2016, MNRAS, 456, 924

DiPompeo, M. A., Myers, A. D., Hickox, R. C., et al. 2015, MNRAS, 446, 3492

DiPompeo, M. A., Myers, A. D., Hickox, R. C., Geach, J. E., \& Hainline, K. N. 2014, MNRAS, 442, 3443

Donley, J. L., Koekemoer, A. M., Brusa, M., et al. 2012, ApJ, 748, 142

Donley, J. L., Rieke, G. H., Pérez-González, P. G., \& Barro, G. 2008, ApJ, 687,111

Donoso, E., Yan, L., Stern, D., \& Assef, R. J. 2014, ApJ, 789, 44

Donoso, E., Yan, L., Tsai, C., et al. 2012, ApJ, 748, 80

Edelson, R., \& Malkan, M. 2012, ApJ, 751, 52

Eisenhardt, P. R. M., Wu, J., Tsai, C.-W., et al. 2012, ApJ, 755, 173

Elvis, M., Wilkes, B. J., McDowell, J. C., et al. 1994, ApJS, 95, 1

Fan, X., Strauss, M. A., Richards, G. T., et al. 2006, AJ, 131, 1203

Fioc, M., \& Rocca-Volmerange, B. 1997, A\&A, 326, 950

Fitzpatrick, E. L. 1999, PASP, 111, 63

Geach, J. E. 2012, MNRAS, 419, 2633

Geach, J. E., Hickox, R. C., Bleem, L. E., et al. 2013, ApJL, 776, L41

Gilli, R., Vignali, C., Mignoli, M., et al. 2010, A\&A, 519, A92

Glikman, E., Simmons, B., Mailly, M., et al. 2015, ApJ, 806, 218

Goulding, A. D., Alexander, D. M., Bauer, F. E., et al. 2012, ApJ, 755, 5

Hainline, K. N., Hickox, R., Greene, J. E., Myers, A. D., \& Zakamska, N. L. 2013, ApJ, 774, 145

Hainline, K. N., Hickox, R. C., Carroll, C. M., et al. 2014a, ApJ, 795, 124

Hainline, K. N., Hickox, R. C., Chen, C.-T., et al. 2016a, ApJ, 823, 42

Hainline, K. N., Hickox, R. C., Greene, J. E., et al. 2014b, ApJ, 787, 65

Hainline, K. N., Reines, A. E., Greene, J. E., \& Stern, D. 2016b, ApJ, 832 119

Hasinger, G., Miyaji, T., \& Schmidt, M. 2005, A\&A, 441, 417

Helfand, D. J., White, R. L., \& Becker, R. H. 2015, ApJ, 801, 26

Hewett, P. C., Warren, S. J., Leggett, S. K., \& Hodgkin, S. T. 2006, MNRAS, 367,454

Hickox, R. C., Jones, C., Forman, W. R., et al. 2007, ApJ, 671, 1365

Hickox, R. C., Jones, C., Forman, W. R., et al. 2009, ApJ, 696, 891

Hickox, R. C., Mullaney, J. R., Alexander, D. M., et al. 2014, ApJ, 782, 9

Hickox, R. C., Myers, A. D., Brodwin, M., et al. 2011, ApJ, 731, 117

Hönig, S. F., Leipski, C., Antonucci, R., \& Haas, M. 2011, ApJ, 736, 26

Hopkins, P. F., \& Hernquist, L. 2009, ApJ, 698, 1550

Hopkins, P. F., Hernquist, L., Cox, T. J., \& Kereš, D. 2008, ApJS, 175, 356

Hopkins, P. F., Hickox, R., Quataert, E., \& Hernquist, L. 2009, MNRAS, 398,333

Husemann, B., Wisotzki, L., Sánchez, S. F., \& Jahnke, K. 2013, A\&A, 549, A43

Hviding, R. E., Hickox, R. C., Hainline, K. N., et al. 2017, MNRAS, submitted Ichikawa, K., Ueda, Y., Terashima, Y., et al. 2012, ApJ, 754, 45

Jarrett, T. H., Cohen, M., Masci, F., et al. 2011, ApJ, 735, 112

Jones, M. L., Hickox, R. C., Black, C. S., et al. 2016, ApJ, 826, 12

Jones, M. L., Hickox, R. C., Mutch, S. J., et al. 2017, ApJ, 843, 125

Kauffmann, G., Heckman, T. M., \& Best, P. N. 2008, MNRAS, 384, 953

Kelly, B. C., Vestergaard, M., Fan, X., et al. 2010, ApJ, 719, 1315

Kewley, L. J., Dopita, M. A., Sutherland, R. S., Heisler, C. A., \& Trevena, J. 2001, ApJ, 556, 121

Kormendy, J., \& Ho, L. C. 2013, ARA\&A, 51, 511

Krumpe, M., Miyaji, T., \& Coil, A. L. 2010, ApJ, 713, 558

Lacy, M., Ridgway, S. E., Gates, E. L., et al. 2013, ApJS, 208, 24

Lacy, M., Ridgway, S. E., Sajina, A., et al. 2015, ApJ, 802, 102

Lacy, M., Storrie-Lombardi, L. J., Sajina, A., et al. 2004, ApJS, 154, 166

LaMassa, S. M., Heckman, T. M., Ptak, A., et al. 2010, ApJ, 720, 786

Lansbury, G. B., Alexander, D. M., Del Moro, A., et al. 2014, ApJ, 785, 17

Lansbury, G. B., Gandhi, P., Alexander, D. M., et al. 2015, ApJ, 809, 115

Lawrence, A., Warren, S. J., Almaini, O., et al. 2007, MNRAS, 379, 1599

Lawrence, A., Warren, S. J., Almaini, O., et al. 2012, VizieR Online Data Catalog: UKIDSS-DR8 LAS, GCS and DXS Surveys, 2314

Martínez-Sansigre, A., Rawlings, S., Lacy, M., et al. 2006, MNRAS, 370, 1479 
Mateos, S., Alonso-Herrero, A., Carrera, F. J., et al. 2012, MNRAS, 426, 3271 McCarthy, P. J. 1993, ARA\&A, 31, 639

Mendez, A. J., Coil, A. L., Aird, J., et al. 2016, ApJ, 821, 55

Mor, R., \& Trakhtenbrot, B. 2011, ApJL, 737, L36

Myers, A. D., Brunner, R. J., Nichol, R. C., et al. 2007, ApJ, 658, 85

Nenkova, M., Sirocky, M. M., Nikutta, R., Ivezić, Ž, \& Elitzur, M. 2008, ApJ, 685,160

Netzer, H. 2015, ARA\&A, 53, 365

Obied, G., Zakamska, N. L., Wylezalek, D., \& Liu, G. 2016, MNRAS, 456, 2861

Padmanabhan, N., White, M., Norberg, P., \& Porciani, C. 2009, MNRAS, 397, 1862

Padovani, P. 2016, A\&ARv, 24, 13

Padovani, P. 2017, NatAs, 1, 0194

Padovani, P., Alexander, D. M., Assef, R. J., et al. 2017, A\&ARv, 25, 2

Page, M. J., Stevens, J. A., Ivison, R. J., \& Carrera, F. J. 2004, ApJL, 611, L85

Plotkin, R. M., Anderson, S. F., Brandt, W. N., et al. 2012, ApJL, 745, L27

Podigachoski, P., Barthel, P., Haas, M., Leipski, C., \& Wilkes, B. 2015, ApJL, 806, L11

Polletta, M. d. C., Wilkes, B. J., Siana, B., et al. 2006, ApJ, 642, 673

Porciani, C., Magliocchetti, M., \& Norberg, P. 2004, MNRAS, 355, 1010

Prandoni, I., Parma, P., Wieringa, M. H., et al. 2006, A\&A, 457, 517

Reyes, R., Zakamska, N. L., Strauss, M. A., et al. 2008, AJ, 136, 2373

Richards, G. T., Croom, S. M., Anderson, S. F., et al. 2005, MNRAS, 360, 839

Richards, G. T., Fan, X., Newberg, H. J., et al. 2002, AJ, 123, 2945

Richards, G. T., Lacy, M., Storrie-Lombardi, L. J., et al. 2006, ApJS, 166, 470

Richards, G. T., Myers, A. D., Gray, A. G., et al. 2009, ApJS, 180, 67

Ross, N. P., Shen, Y., Strauss, M. A., et al. 2009, ApJ, 697, 1634

Rowan-Robinson, M., Babbedge, T., Surace, J., et al. 2005, AJ, 129, 1183

Sajina, A., Yan, L., Fadda, D., Dasyra, K., \& Huynh, M. 2012, ApJ, 757, 13

Sanders, D. B., Soifer, B. T., Elias, J. H., et al. 1988, ApJ, 325, 74

Schaye, J., Crain, R. A., Bower, R. G., et al. 2015, MNRAS, 446, 521
Secrest, N. J., Dudik, R. P., Dorland, B. N., et al. 2015, ApJS, 221, 12 Setti, G., \& Woltjer, L. 1989, A\&A, 224, L21

Seymour, N., Stern, D., De Breuck, C., et al. 2007, ApJS, 171, 353

Shen, Y., Richards, G. T., Strauss, M. A., et al. 2011, ApJS, 194, 45

Shen, Y., Strauss, M. A., Oguri, M., et al. 2007, AJ, 133, 2222

Sikora, M., Stawarz, Ł., \& Lasota, J.-P. 2007, ApJ, 658, 815

Skrutskie, M. F., Cutri, R. M., Stiening, R., et al. 2006, AJ, 131, 1163

Stalevski, M., Ricci, C., Ueda, Y., et al. 2016, MNRAS, 458, 2288

Stern, D., Assef, R. J., Benford, D. J., et al. 2012, ApJ, 753, 30

Stern, D., Eisenhardt, P., Gorjian, V., et al. 2005, ApJ, 631, 163

Stern, D., Lansbury, G. B., Assef, R. J., et al. 2014, ApJ, 794, 102

Stern, D., Moran, E. C., Coil, A. L., et al. 2002, ApJ, 568, 71

Tadhunter, C. 2016, A\&ARv, 24, 10

Taylor, M. B. 2005, in ASP Conf. Ser. 347, Astronomical Data Analysis Software and Systems XIV, ed. P. Shopbell, M. Britton, \& R. Ebert (San Francisco, CA: ASP), 29

Thacker, R. J., MacMackin, C., Wurster, J., \& Hobbs, A. 2014, MNRAS, 443, 1125

Tinker, J. L., \& Wetzel, A. R. 2010, ApJ, 719, 88

Treister, E., Urry, C. M., Chatzichristou, E., et al. 2004, ApJ, 616, 123

Urry, C. M., \& Padovani, P. 1995, PASP, 107, 803

Vignali, C., Alexander, D. M., \& Comastri, A. 2006, MNRAS, 373, 321

Vignali, C., Pozzi, F., Fritz, J., et al. 2009, MNRAS, 395, 2189

Vogelsberger, M., Genel, S., Springel, V., et al. 2014, Natur, 509, 177

Wilkes, B. J., Kuraszkiewicz, J., Haas, M., et al. 2013, ApJ, 773, 15

Wright, E. L., Eisenhardt, P. R. M., Mainzer, A. K., et al. 2010, AJ, 140, 1868

Yuan, S., Strauss, M. A., \& Zakamska, N. L. 2016, MNRAS, 462, 1603

Zakamska, N. L., Strauss, M. A., Heckman, T. M., Ivezić, Ž, \& Krolik, J. H. 2004, AJ, 128, 1002

Zakamska, N. L., Strauss, M. A., Krolik, J. H., et al. 2003, AJ, 126, 2125

Zakamska, N. L., Strauss, M. A., Krolik, J. H., et al. 2005, AJ, 129, 1212

Zakamska, N. L., Strauss, M. A., Krolik, J. H., et al. 2006, AJ, 132, 1496 